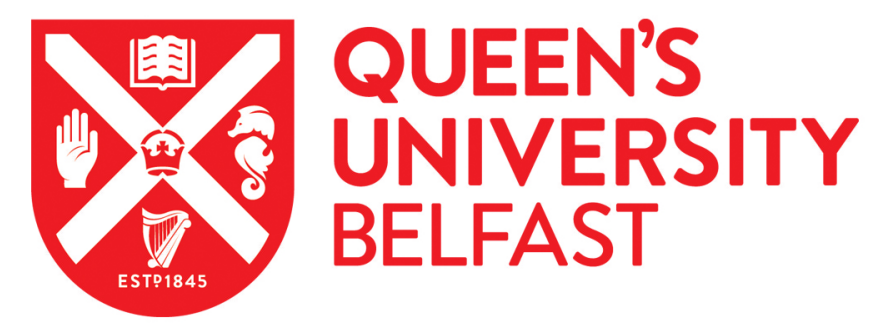

\title{
Joint D2D Assignment, Bandwidth and Power Allocation in Cognitive UAV-enabled Networks
}

Nguyen, H. T., Duong, T. Q., \& Tuan, H. D. (2020). Joint D2D Assignment, Bandwidth and Power Allocation in Cognitive UAV-enabled Networks. IEEE Transactions on Cognitive Communications and Networking.

https://doi.org/10.1109/TCCN.2020.2969623

Published in:

IEEE Transactions on Cognitive Communications and Networking

Document Version:

Peer reviewed version

Queen's University Belfast - Research Portal:

Link to publication record in Queen's University Belfast Research Portal

Publisher rights

(c) 2020 IEEE.

This work is made available online in accordance with the publisher's policies. Please refer to any applicable terms of use of the publisher.

\section{General rights}

Copyright for the publications made accessible via the Queen's University Belfast Research Portal is retained by the author(s) and / or other copyright owners and it is a condition of accessing these publications that users recognise and abide by the legal requirements associated with these rights.

Take down policy

The Research Portal is Queen's institutional repository that provides access to Queen's research output. Every effort has been made to ensure that content in the Research Portal does not infringe any person's rights, or applicable UK laws. If you discover content in the Research Portal that you believe breaches copyright or violates any law, please contact openaccess@qub.ac.uk. 


\title{
Joint D2D Assignment, Bandwidth and Power Allocation in Cognitive UAV-enabled Networks
}

\author{
H. T. Nguyen, H. D. Tuan, T. Q. Duong, H. V. Poor, and W-J. Hwang
}

\begin{abstract}
This paper considers a cognitive communication network, which consists of a flying base station deployed by an unmanned aerial vehicle (UAV) to serve its multiple downlink ground terminals (GTs), and multiple underlaid device-to-device (D2D) users. To support the GTs' throughput while guaranteeing the quality-of-service for the D2D users, the paper proposes the joint design of D2D assignment, bandwidth, and power allocation. This design task poses a computationally challenging mixedbinary optimization problem, for which a new computational method for its solution is developed. Multiple binary (discrete) constraints for the D2D assignment are equivalently expressed by continuous constraints to leverage systematic processes of continuous optimization. As a result, this problem of mixedbinary optimization is reformulated by an exactly penalized continuous optimization problem, for which an alternating descent algorithm is proposed. Each round of the algorithm invokes two simple convex optimization problems of low computational complexity. The theoretical convergence of the algorithm can be easily proved and the provided numerical results demonstrate its rapid convergence to an optimal solution. Such a cognitive network is even more desirable as it outperforms a non-cognitive network, which uses a partial bandwidth for D2D users only.
\end{abstract}

Index Terms-Unmanned aerial vehicle (UAV)-enabled network, cognitive device-to-device (D2D) communication, bandwidth allocation, power allocation, D2D assignment, mixedinteger programming

\section{INTRODUCTION}

Using an unmanned aerial vehicle (UAV) as a flying base station promises to support the coverage and throughput of wireless communications [1]. In contrast to conventional cellular communication, which is shadowed by non-line-of-sight (NLoS) connections, such UAV-enabled communication leverages the strength of line-of-sight (LoS) connections [2]. The air-to-ground channel model is not diffracted or reflected much

This work was supported in part by the Institute for Computational Science and Technology, Hochiminh City, Vietnam, in part by the Australian Research Councils' Discovery Projects under Project DP190102501, in part by the U.K. Royal Academy of Engineering Research Fellowship under Grant RF1415 \14\22, in part by the U.S. National Science Foundation under Grants under Grants CCF-0939370 and CCF-1908308.

Huy T. Nguyen is now with School of Computer Science and Engineering, Nanyang Technological University, Singapore (email: huyt.nguyen@ntu.edu.sg).

Hoang Duong Tuan is with the School of Electrical and Data Engineering, University of Technology Sydney, Broadway, NSW 2007, Australia (email: tuan.hoang@uts.edu.au).

Trung Q. Duong is with the School of Electronics, Electrical Engineering and Computer Science, Queen's University Belfast, Belfast BT7 1NN, U.K. (email: trung.q.duong@qub.ac.uk).

H. Vincent Poor is with the Department of Electrical Engineering, Princeton University, Princeton, NJ 08544, USA (email: poor@princeton.edu).

Won-Joo Hwang is with the School of Biomedical Convergence Engineering, Pusan National University, Gyeongsangnam-do 50612, Korea (email: wjhwang@pusan.ac.kr). by obstructions compared to the ground-to-ground channel model. Therefore, UAV-enabled communication can achieve a high throughput at ground terminals (GTs) even under a long range communication circumstance. The deployment of UAVenabled networks not only is cost-saving but also resolves occupancy, which is a serious issue in urban areas. UAVenabled communications has found interesting applications in military, emergency missions, and outdoor events [3]-[5], where UAVs can act as temporary hotspots or relay nodes to establish connections, for example, between the safety area and disaster areas [6], [7].

To realize the benefits of UAV-enabled communication in terms of coverage area, throughput, and energy efficiency, many typical problems such as UAV placement [8]-[10], UAV trajectory design [11], [12], beamwidth control [13], [14], and power allocation [15]-[18] have been studied. Under the coexistence of secondary networks, the effect of NLoS and LoS connections to the GT throughput has been analyzed in [16], [19], [20].

In recent years, device-to-device (D2D) communication [21]-[25] to support various high-speed services without having to install any essential infrastructures has emerged as an important feature in the 5th generation of cellular networks (5G) [26]. In the previous studies such as [27], [28], the pairs in close proximity are directly linked as cognitive users or primary users. Cognitive D2D communication, in which D2D users reuse resources of the cellular communication systems has received significant attention [26], [29], [30]. The objective is interference management to guarantee the qualityof-service (QoS) requirements for both (primary) cellular users and (cognitive) D2D users [31]-[33].

In contrast to the well-studied conventional D2D cognitive cellular networks, the viability of D2D cognitive UAVenabled networks remains mostly unknown. Their coverage and throughput have been analyzed in [34]. The so called d.c. (difference of two convex) iterations [35] are used in [36] to maximize sum rate subject to the QoS requirements for both GT and D2D users, who use the whole communication bandwidth at the same time. Each d.c. iteration involves maximization of a logarithmic function, which is although a convex problem but there is no solver of polynomial complexity. A cognitive network of multiple primary GT users and a single cognitive D2D user was considered in [37], where the bandwidth allocation is only applied to the GT users while the D2D user uses the whole bandwidth, and thus it suffers severe interference from all the GT users. It should be noted that the information theoretic analysis of the conventional D2D cognitive cellular networks is based on the ground-to- 
ground fading model, where the connections between the base station and users are NLoS. In contrast, the strong air-toground fading is presented in the interference channels from UAV to D2D users in D2D cognitive UAV-enabled networks. As such, the throughput of cognitive D2D communication is limited by LoS interference links. Therefore, new strategies of interference management are essential to ensure the quality-ofservice (QoS) in terms of D2D throughput. To the best of the authors' knowledge, there were no previous studies concerning with the GT throughput enhancement while guaranteeing the QoS in terms of D2D throughput. The main contributions of this paper are summarized as follows.

- For a cognitive communication network, which consists of a UAV deployed as a flying base station to serve multiple GTs and multiple underlaid D2D users, the paper proposes a new joint design of D2D assignment, bandwidth, and power allocation in order to maximize the minimum GT throughput subject to the QoS requirement for the D2D users.

- Such a design task poses a complex mixed-integer optimization problem, which needs a new computational solution. The paper introduces a novel function to measure the degree of satisfaction of the binary constraints, which helps to recast this mixed-integer optimization problem by a new exactly penalized optimization problem of continuous optimization. An alternating descent algorithm is then developed for its computation. Each round of the algorithm undergoes two iterations for alternating optimization in D2D assignment, bandwidth and power allocations. Each iteration invokes a simple convex optimization of low computational complexity. The theoretical convergence of the algorithms can be easily proved, while their rapid convergence is supported by numerical results.

- Such a cognitive communication network is shown to perform best when the UAV hovers at the lowest possible altitude. This is very desirable because it is well-known [38] that the UAV consumes the lowest energy when hovering but not when moving or circling.

- Such a cognitive communication is also shown to outperform a non-cognitive network, which uses a partial bandwidth for D2D users to completely avoid LoS interference links. Therefore, the cognitive LoS interference enhancement instead of the non-cognitive LoS interference rejection is well motivated.

The rest of this paper is organized as follows. The cognitive system model and problem formulations are introduced in Section II. An alternating descent algorithm for its computation is developed in Section III. Section IV extends the GT throughput max-min problem in a non-cognitive system, which allocates a partial bandwidth to D2D users for the full UAV interference rejection. Numerical results are presented in Section V and conclusions are drawn in Section VI.

Notation. Boldface symbols are used for optimization variables. For $x=\left(x_{1}, \ldots, x_{n}\right)^{T} \in \mathbb{R}^{n}, x \geq 0$ and $x^{2}$ are entrywise understood, i.e., $x \geq 0$ means $x_{i} \geq 0, i=1, \ldots, n$ and $x^{2} \triangleq\left(x_{1}^{2}, \ldots, x_{n}^{2}\right)$.

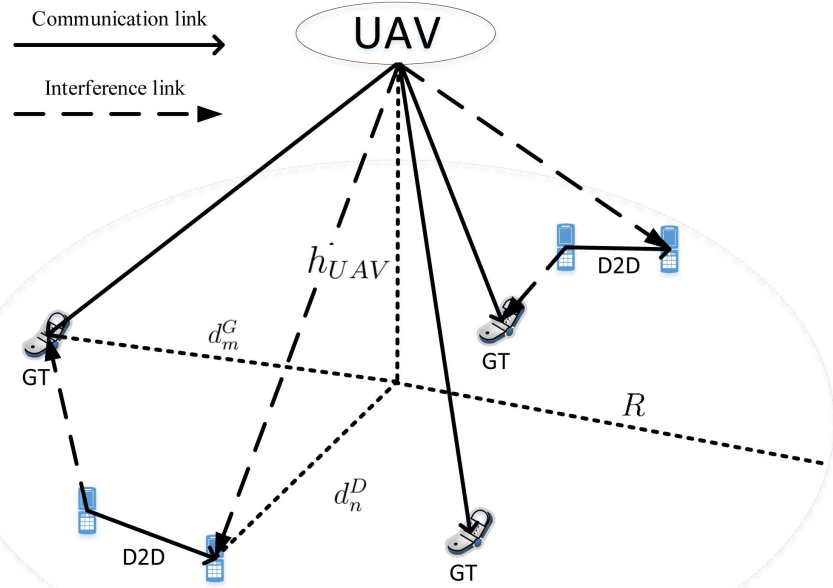

Fig. 1. The downlink scenario of UAV-enabled networks coexisting with D2D communication, where the UAV is deployed at the center of the virtual cell.

The following inequalities [39] for all $x>0, y>0, t>0$ and $\bar{x}>0, \bar{y}>0, \bar{t}>0$ will be frequently used in the paper:

$$
\ln \left(1+\frac{1}{x}\right) \geq \ln \left(1+\frac{1}{\bar{x}}\right)+\frac{1}{\bar{x}+1}\left(1-\frac{x}{\bar{x}}\right)
$$

and

$$
\begin{aligned}
y \ln \left(1+\frac{1}{x}\right) \geq & 2 \bar{y} \ln \left(1+\frac{1}{\bar{x}}\right)+\frac{\bar{y}}{\bar{x}+1}\left(1-\frac{x}{\bar{x}}\right) \\
& -\bar{y}^{2} \frac{\ln (1+1 / \bar{x})}{y},
\end{aligned}
$$

and

$$
\ln \left(1+\frac{1}{x y}\right) \geq \ln \left(1+\frac{1}{\bar{x} \bar{y}}\right)+\frac{1 / \bar{x} \bar{y}}{1+1 / \bar{x} \bar{y}}\left(2-\frac{x}{\bar{x}}-\frac{y}{\bar{y}}\right)
$$

and

$$
\begin{aligned}
\frac{\ln (1+1 / x y)}{t} \geq & \frac{2 \ln (1+1 / \bar{x} \bar{y})}{\bar{t}}+\frac{1 / \bar{x} \bar{y}}{\bar{t}(1+1 / \bar{x} \bar{y})} \\
& \times\left(2-\frac{x}{\bar{x}}-\frac{y}{\bar{y}}\right)-\frac{\ln (1+1 / \bar{x} \bar{y})}{\bar{t}^{2}} t .
\end{aligned}
$$

\section{COGNITIVE SYSTEM MODEL \& PROBLEM FORMULATIONS}

\section{A. System model}

Consider a downlink UAV-enabled network as illustrated by Fig. 1, which consists of a UAV deployed at the center of the virtual cell with radius $R$ to transfer information to the set of $M$ ground terminals (GTs) $G T_{m}, m \in \mathcal{M} \triangleq\{1, \ldots, M\}$. The GTs are randomly located in the area. There is a set of $N$ device-to-device (D2D) pairs $D_{n}, n \in \mathcal{N} \triangleq\{1, \ldots, N\}$, which are allowed to use the same spectrum of the UAVenabled communication. Assuming that all GTs and D2Ds are located outdoors in rural areas, the communication link between the UAV and GTs and the UAV interference to D2Ds are dominated by LoS air-to-ground channels. The channel 
power gain for the LoS path between the UAV and $G T_{m}$, named $h_{m}^{U G}$, is given by [13]

$$
h_{m}^{U G}=\omega_{0} /\left(\sqrt{h_{U A V}^{2}+\left(d_{m}^{G}\right)^{2}}\right)^{\alpha_{L o S}},
$$

where $\omega_{0}=\left(4 \pi f_{c} / c\right)^{-2}$ is the channel power gain at the unit reference distance (one meter) under the carrier frequency $f_{c}$ and the speed of light $c, h_{U A V}$ is the altitude of the UAV, $d_{m}^{G}$ $\left(d_{m}^{G} \leq R\right)$ is the horizontal distance from the UAV to $G T_{m}$, while $\alpha_{L o S} \geq 2$ is the LoS path-loss exponent. ${ }^{1}$ Similarly, the channel power gain for LoS path between the UAV and $D_{n}$, named $g_{n}^{U D}$, is given by

$$
g_{n}^{U D}=\omega_{0} /\left(\sqrt{h_{U A V}^{2}+\left(d_{n}^{D}\right)^{2}}\right)^{\alpha_{L o S}},
$$

where $d_{n}^{D}$ is the horizontal distance between the UAV and $D_{n}$ receiver, which is not more than $R$. Assuming that orthogonal frequency bands are allocated to the multiple GTs, each D2D pair must select one from GTs' frequency bands for its communication. Define the indicator

$$
\mathbf{x}_{n, m}= \begin{cases}1 & \text { if } D_{n} \text { shares the bandwidth with } G T_{m} \\ 0 & \text { otherwise. }\end{cases}
$$

Suppose $\mathbf{w}_{m}$ is the allocated bandwidth to $G T_{m}, \mathbf{p}_{m}^{U}$ is the power of signal carrying the information intended for $G T_{m}$, and $\mathbf{p}_{n}^{D}$ is the transmit power of $D_{n}$. Further, $h_{n}^{D}$ is the direct communication channel for the $n$-th D2D pair, $g_{n, m}^{D G}$ is the interference channel between $D_{n}$ transmitter and the $G T_{m}$, and $g_{j, n}^{D}$ is the interference channel between $D_{j}$ transmitter and $D_{n}$ receiver, which are modeled by NLoS channels.

For

$$
\begin{gathered}
\mathbf{x}_{m} \triangleq\left(\mathbf{x}_{n, m}\right)_{n \in \mathcal{N}}, \mathbf{x} \triangleq\left(\mathbf{x}_{m}\right)_{m \in \mathcal{M}}, \mathbf{w} \triangleq\left(\mathbf{w}_{m}\right)_{m \in \mathcal{M}}, \\
\mathbf{p}^{U} \triangleq\left(\mathbf{p}_{m}^{U}\right)_{m \in \mathcal{M}}, \mathbf{p}^{D} \triangleq\left(\mathbf{p}_{n}^{D}\right)_{n \in \mathcal{N}}, \mathbf{p}=\left(\mathbf{p}^{U}, \mathbf{p}^{D}\right),
\end{gathered}
$$

the throughput at $G T_{m}$ and $D_{n}$ can be expressed as

$$
\begin{aligned}
& r_{m}^{G}(\mathbf{x}, \mathbf{w}, \mathbf{p}) \\
& =\mathbf{w}_{m} \ln \left(1+\frac{\mathbf{p}_{m}^{U} h_{m}^{U G}}{\sum_{n=1}^{N} \mathbf{x}_{n, m} \mathbf{p}_{n}^{D} g_{n, m}^{D G}+\mathbf{w}_{m} \sigma_{m}^{2}}\right),
\end{aligned}
$$

and

$$
\begin{aligned}
& r_{n}^{D}(\mathbf{x}, \mathbf{w}, \mathbf{p}) \\
& =\sum_{m=1}^{M} \mathbf{w}_{m} \mathbf{x}_{n, m} \\
& \quad \times \ln \left(1+\frac{\mathbf{p}_{n}^{D} h_{n}^{D}}{\mathbf{p}_{m}^{U} g_{n}^{U D}+\sum_{j \in \mathcal{N} \backslash\{n\}} \mathbf{x}_{j, m} \mathbf{p}_{j}^{D} g_{j, n}^{D}+\mathbf{w}_{m} \sigma_{n}^{2}}\right) .
\end{aligned}
$$

${ }^{1}$ Like [13] $\alpha_{\text {LoS }}=2$ is set in this paper

\section{B. Exactly penalized optimization reformulation}

Our objective is to maximize the minimum throughput among GTs subject to transmit power constraints on the UAV and D2D pairs, and quality-of-service (QoS) for D2D in terms of its throughput by addressing the following mixed-binary optimization problem:

$$
\begin{array}{ll} 
& \max _{\mathbf{x}, \mathbf{w}, \mathbf{p}} f(\mathbf{x}, \mathbf{w}, \mathbf{p}) \triangleq \min _{m \in \mathcal{M}} r_{m}^{G}(\mathbf{x}, \mathbf{w}, \mathbf{p}) \\
\text { s.t. } & \mathbf{w}_{m} \geq 0, m \in \mathcal{M}, \\
& \sum_{m=1}^{M} \mathbf{w}_{m}=\mathcal{B}, \\
& \mathbf{x} \in\{0,1\}^{N M}, \\
& \sum_{m=1}^{M} \mathbf{x}_{n, m}=1, n \in \mathcal{N}, \\
& \sum_{m=1}^{M} \mathbf{p}_{m}^{U} \leq P_{\max }^{U}, \\
& 0<\mathbf{p}_{n}^{D} \leq P_{\max }^{D}, n \in \mathcal{N}, \\
& r_{n}^{D}(\mathbf{x}, \mathbf{w}, \mathbf{p}) \geq r_{\min }^{D}, n \in \mathcal{N} .
\end{array}
$$

The constraint (9c) indicates that the given bandwidth $\mathcal{B}$ is divided for all GTs. Constraint (9e) guarantees that each D2D pair reuses the spectrum of one GT only. Constraints (9f) and $(9 \mathrm{~g})$ represent the maximum transmit power constraints at the UAV and D2D transmitter, respectively. Finally, constraint (9h) ensures the QoS requirement in terms of throughput in the D2D communication.

As mentioned in the Introduction, the work [34] is interested in analysing the network coverage and throughput so it does not aim to solve any optimization problem. On the other hand, there is only a single cognitive D2D user in [37], who shares the bandwidth with all GTs, so there is no need to assign the D2D user to a particular GT for sharing the bandwidth. The corresponding optimization problem is not combinatoric and thus is much easier for computation.

To clarify the computational difficulty of the above mixedbinary optimization problem, it is worth noticing that (9) is still a difficult nonconvex problem in $(\mathbf{w}, \mathbf{p})$ of resource when the binary variable $\mathbf{x}$ of assignment is held fixed and it is still a difficult combinatoric optimization in the binary variable $\mathbf{x}$ when the continuous variables $\mathbf{w}$ and $\mathbf{p}$ are held fixed. A branch-and-bound algorithm of global optimization [40] has been used in [32] for a combinatoric optimization problem of assignment, which is not practical for moderate numbers of D2D users. Power optimization for the conventional D2D underlaid cellular networks has been addressed in [33] by d.c. iterations [35]. Furthermore, a much more efficient power optimization algorithm, which avoids logarithmic function optimization of non tractable computation, has been recently proposed in [39].

Our previous works [41] and [42] have demonstrated that the exactly penalized optimization, which simultaneously maximizes the objective function (9a) and the degree of satisfaction of the binary constraints (9d), is appropriate for addressing the mixed-binary optimization problem (9). We now provide a novel exactly penalized optimization re-formulation 
of continuous optimization for the mixed-binary optimization problem (9), which particularly involves a new continuous reformulation of the binary constraints (9d) and also a new measure of binary satisfaction.

First of all, the binary constraint (9d) and linear equality constraint $(9 \mathrm{e})$ are equivalent to the linear equality constraint (9e) and the box constraint

$$
\mathbf{x} \in[0,1]^{N M}
$$

and the constraint

$$
\frac{1}{g(\mathbf{x})} \leq \frac{1}{N}
$$

with

$$
g(\mathbf{x})=\sum_{n=1}^{N} \sum_{m=1}^{M} \mathbf{x}_{n, m}^{L}
$$

for $L>1$. Indeed, as $\mathbf{x}_{n, m} \in[0,1]$ it follows that $\mathbf{x}_{n, m}^{L}<$ $\mathbf{x}_{n, m}$ for $\mathbf{x}_{n, m} \in(0,1)$ and $\mathbf{x}_{n, m}^{L}=\mathbf{x}_{n, m}$ for $\mathbf{x}_{n, m} \in\{0,1\}$. Therefore,

$$
\frac{1}{g(\mathbf{x})} \geq \frac{1}{\sum_{n=1}^{N} \sum_{m=1}^{M} \mathbf{x}_{n, m}}=\frac{1}{N},
$$

and $1 / g(\mathbf{x})=1 / N$ if and only if $\mathbf{x}_{n, m} \in\{0,1\} \forall(n, m) \in$ $\mathcal{N} \times \mathcal{M}$. The constraint (11) thus forces all $\mathbf{x}_{n, m}$ to binary values. Therefore, the function

$$
\widetilde{g}(\mathbf{x})=\frac{1}{N}-\frac{1}{g(\mathbf{x})}
$$

can be used to measure the degree of satisfaction of the binary constraint (9d) and linear equality constraint (9e) in the sense that $\widetilde{g}(\mathbf{x}) \leq 0$ for $\mathbf{x} \in[0,1]^{N M}$ and $\widetilde{g}(\mathbf{x})=0$ if and only if $\mathbf{x} \in\{0,1\}^{N M}$.

Following our previous developments in [41], [42], instead of handling constraint (11), we incorporate the degree of its satisfaction into the objective in (9), leading to the following penalized optimization problem:

$$
\begin{array}{r}
\max _{\mathbf{x}, \mathbf{w}, \mathbf{p}} f_{\mu}(\mathbf{x}, \mathbf{w}, \mathbf{p}) \triangleq f\left(\mathbf{x}^{2}, \mathbf{w}, \mathbf{p}\right)+\mu \widetilde{g}(\mathbf{x}) \\
\text { s.t. } \quad(9 b)-(9 c),(9 e)-(9 h),(10),
\end{array}
$$

where $\mu>0$ is a penalty parameter. This penalized optimization problem is exact with a sufficiently large $\mu$ in the sense that (14) and (9) share the same optimal solution. Note that we have placed the variable $\mathrm{x}^{2}$ in the place of $\mathrm{x}$ in function $f$ because $\mathbf{x}^{2}=\mathbf{x}$ for $\mathbf{x} \in\{0,1\}^{N M}$.

Usually, $\mu$ is found from the beginning as follows. Taking any feasible point $x^{(0)}$ for (9e), (10), iterate

$$
\max _{\mathbf{w}, \mathbf{p}} \min \left\{\min _{m \in \mathcal{M}} \frac{r_{m}^{G}\left(x^{(0)}, \mathbf{w}, \mathbf{p}\right)}{r_{\min }^{U}}, \min _{n \in \mathcal{N}} \frac{r_{n}^{D}\left(x^{(0)}, \mathbf{w}, \mathbf{p}\right)}{r_{\min }^{D}}\right\}
$$$$
\text { s.t. }(9 b)-(9 c),(9 f)-(9 g) \text {, }
$$

till the objective value is more than equal to one at $\left(w^{*}, p^{*}\right)$. Thus $\left(x^{(0)}, w^{*}, p^{*}\right)$ is a feasible point for (14). Here $r_{\min }^{U}$ is chosen beforehand to have a reasonable value $\min _{m \in \mathcal{M}} r_{m}^{G}\left(x^{(0)}, w^{*}, p^{*}\right)$ for initializing the computational procedure described in the next subsections. We also describe iterations for (15) in a later subsection.
Then set $\left(x^{(0)}, w^{(0)}, p^{(0)}\right)=\left(x^{(0)}, w^{*}, p^{*}\right)$ and

$$
\mu=f\left(x^{(0)}, w^{(0)}, p^{(0)}\right) /\left(-\widetilde{g}\left(x^{(0)}\right)\right) .
$$

Let $\left(x^{(\kappa)}, w^{(\kappa)}, p^{(\kappa)}\right)$ be a feasible point for (14) found from the $\kappa$-th iteration. Next subsections provide the next $(\kappa+1)$-th iteration to generate the next iterative feasible point $\left(x^{(\kappa+1)}, w^{(\kappa+1)}, p^{(\kappa+1)}\right)$ for (14).

\section{Alternating Descent Algorithm for Maximin GT RATE OPTIMIZATION}

\section{A. D2D assignment optimization}

For $(\mathbf{w}, \mathbf{p})$ held fixed at $(w, p) \triangleq\left(w^{(\kappa)}, p^{(\kappa)}\right)$ we consider problem (14) in variable $\mathbf{x}$ :

$$
\begin{array}{ll} 
& \max _{\mathbf{x}} f_{1}(\mathbf{x}) \triangleq \min _{m \in \mathcal{M}} r_{m}^{G}\left(\mathbf{x}^{2}, w, p\right)+\mu \widetilde{g}(\mathbf{x}) \\
\text { s.t } & (9 e),(10), \\
& r_{n}^{D}(\mathbf{x}, w, p) \geq r_{\min }^{D}, n \in \mathcal{N},
\end{array}
$$

which is a difficult nonconvex optimization problem as both functions $r_{m}^{G}\left(\mathbf{x}^{2}, w, p\right)$ and $\widetilde{g}(\mathbf{x})$, making the objective function in (17a) nonconcave, and constraint (17b) is nonconvex.

Applying inequality (1) in Section I for

$$
x=\frac{\sum_{n=1}^{N} \mathbf{x}_{n, m} p_{n}^{D} g_{n, m}^{D G}+w_{m} \sigma_{m}^{2}}{p_{m}^{U} h_{m}^{U G}}
$$

and

$$
\bar{x}=\frac{\sum_{n=1}^{N} x_{n, m}^{(\kappa)} p_{n}^{D} g_{n, m}^{D G}+w_{m} \sigma_{m}^{2}}{p_{m}^{U} h_{m}^{U G}}
$$

yields

$$
\begin{aligned}
r_{m}^{G}\left(\mathbf{x}^{2}, w, p\right) \geq & a_{m}^{(\kappa)}+b_{m}^{(\kappa)} \\
& \times \frac{\sum_{n=1}^{N}\left[\left(x_{n, m}^{(\kappa)}\right)^{2}-\left(\mathbf{x}_{n, m}\right)^{2}\right] p_{n}^{D} g_{n, m}^{D G}}{\sum_{n=1}^{N}\left(x_{n, m}^{(\kappa)}\right)^{2} p_{n}^{D} g_{n, m}^{D G}+w_{m} \sigma_{m}^{2}} \\
\triangleq & r_{m}^{G(\kappa)}\left(\mathbf{x}^{2}, w, p\right),
\end{aligned}
$$

where

$$
\begin{aligned}
a_{m}^{(\kappa)} & =w_{m} \ln \left(1+\frac{p_{m}^{U} h_{m}^{U G}}{\sum_{n=1}^{N}\left(x_{n, m}^{(\kappa)}\right)^{2} p_{n}^{D} g_{n, m}^{D G}+w_{m} \sigma_{m}^{2}}\right) \\
b_{m}^{(\kappa)} & =\frac{w_{m}}{1+\left(\sum_{n=1}^{N}\left(x_{n, m}^{(\kappa)}\right)^{2} p_{n}^{D} g_{n, m}^{D G}+w_{m} \sigma_{m}^{2}\right) / p_{m}^{U} h_{m}^{U G}} .
\end{aligned}
$$

Next, each function $\mathbf{x}_{n, m}^{L}$ is convex in $\mathbf{x}_{n, m}>0$ because its derivative $\left(\mathbf{x}_{n, m}^{L}\right)^{\prime}=L \mathbf{x}_{n, m}^{L-1}$ is an increasing function [40]. Therefore function $g(\mathbf{x})$ defined by (12) is convex. This means that for all $\mathbf{x} \geq 0$ and $x^{(\kappa)} \geq 0$, the following inequality is valid

$$
\begin{aligned}
g(\mathbf{x}) & \geq g^{(\kappa)}(\mathbf{x}) \\
& \triangleq g\left(x^{(\kappa)}\right)+\left\langle\nabla g\left(x^{(\kappa)}\right), \mathbf{x}-x^{(\kappa)}\right\rangle \\
& =\sum_{n=1}^{N} \sum_{m=1}^{M}\left(L\left(x_{n, m}^{(\kappa)}\right)^{L-1} \mathbf{x}_{n, m}-(L-1)\left(x_{n, m}^{(\kappa)}\right)^{L}\right) .
\end{aligned}
$$


On the other hand,

$$
\widetilde{g}(\mathbf{x}) \geq \frac{1}{N}-\frac{1}{g^{(\kappa)}(\mathbf{x})}
$$

over the trust region

$$
g^{(\kappa)}(\mathbf{x})>0
$$

where both $g^{(\kappa)}(\mathbf{x})$ and $1 / g^{(\kappa)}(\mathbf{x})$ are convex functions. Therefore,

$$
\begin{aligned}
f_{1}(\mathbf{x}) & \geq f_{1}^{(\kappa)}(\mathbf{x}, w, p) \\
& \triangleq \min _{m \in \mathcal{M}} r_{m}^{G(\kappa)}\left(\mathbf{x}^{2}, w, p\right)+\mu\left(\frac{1}{N}-\frac{1}{g^{(\kappa)}(\mathbf{x})}\right) .
\end{aligned}
$$

Function $f_{1}^{(\kappa)}(\mathbf{x}, w, p)$ is seen concave because the first term in (25) is concave as minimum of concave functions [40] and the second term is already concave. Also, it can be immediately checked that

$$
f_{1}\left(x^{(\kappa)}\right)=f_{1}^{(\kappa)}\left(x^{(\kappa)}, w, p\right)
$$

Applying inequality (1) in Section I again for

$$
x=\frac{p_{m}^{U} g_{n}^{U D}+\sum_{j \in \mathcal{N} \backslash\{n\}} \mathbf{x}_{j, m} p_{j}^{D} g_{j, n}^{D}+w_{m} \sigma_{n}^{2}}{p_{n}^{D} h_{n}^{D}}
$$

and

$$
\bar{x}=\frac{p_{m}^{U} g_{n}^{U D}+\sum_{j \in \mathcal{N} \backslash\{n\}} x_{j, m}^{(\kappa)} p_{j}^{D} g_{j, n}^{D}+w_{m} \sigma_{n}^{2}}{p_{n}^{D} h_{n}^{D}}
$$

we obtain

$$
\begin{aligned}
r_{n}^{D}(\mathbf{x}, w, p) \geq & \sum_{m=1}^{M} \mathbf{x}_{n, m}\left[\alpha_{n, m}^{(\kappa)}+\beta_{n, m}^{(\kappa)}\right. \\
& \left.\times \frac{\sum_{j \in \mathcal{N} \backslash\{n\}}\left(x_{j, m}^{(\kappa)}-\mathbf{x}_{j, m}\right) p_{j}^{D} g_{j, n}^{D}}{p_{m}^{U} g_{n}^{U D}+\sum_{j \in \mathcal{N} \backslash\{n\}} x_{j, m}^{(\kappa)} p_{j}^{D} g_{j, n}^{D}+w_{m} \sigma_{n}^{2}}\right],
\end{aligned}
$$

where

$$
\begin{aligned}
0<\alpha_{n, m}^{(\kappa)} & \\
& =w_{m} \ln \left(1+\frac{p_{n}^{D} h_{n}^{D}}{p_{m}^{U} g_{n}^{U D}+\sum_{j \in \mathcal{N} \backslash\{n\}} x_{j, m}^{(\kappa)} p_{j}^{D} g_{j, n}^{D}+w_{m} \sigma_{n}^{2}}\right),
\end{aligned}
$$

$$
\begin{aligned}
0 & <\beta_{n, m}^{(\kappa)} \\
& =\frac{w_{m}}{1+\left(p_{m}^{U} g_{n}^{U D}+\sum_{j \in \mathcal{N} \backslash\{n\}} x_{j, m}^{(\kappa)} p_{j}^{D} g_{j, n}^{D}+w_{m} \sigma_{n}^{2}\right) / p_{n}^{D} h_{n}^{D}} .
\end{aligned}
$$

For

$$
\begin{aligned}
& 0<\chi_{n, m}^{(\kappa)} \triangleq \\
& \alpha_{n, m}^{(\kappa)}+\frac{\beta_{n, m}^{(\kappa)} \sum_{j \in \mathcal{N} \backslash\{n\}} x_{j, m}^{(\kappa)} p_{j}^{D} g_{j, n}^{D}}{p_{m}^{U} g_{n}^{U D}+\sum_{j \in \mathcal{N} \backslash\{n\}} x_{j, m}^{(\kappa)} p_{j}^{D} g_{j, n}^{D}+w_{m} \sigma_{n}^{2}}, \\
& 0<\delta_{n, m}^{(\kappa)} \triangleq \frac{\beta_{n, m}^{(\kappa)}}{p_{m}^{U} g_{n}^{U D}+\sum_{j \in \mathcal{N} \backslash\{n\}} x_{j, m}^{(\kappa)} p_{j}^{D} g_{j, n}^{D}+w_{m} \sigma_{n}^{2}},
\end{aligned}
$$

rewrite the right hand side (RHS) of (27) as (30) in the top of next page, over the trust region

$$
r_{n}^{D(\kappa)}(\mathbf{x}, w, p)>0, n \in \mathcal{N},
$$

which is convex because functions $r_{n}^{D(\kappa)}$ are concave. The nonconvex constraint (17b) is then innerly approximated by the convex constraint

$$
r_{n}^{D(\kappa)}(\mathbf{x}, w, p) \geq r_{\text {min }}^{D}, n \in \mathcal{N},
$$

because the feasibility set of the latter is a subset of the feasibility set of the former.

We solve the following convex optimization problem to generate the next iterative point $x^{(\kappa+1)}$ :

$$
\max _{\mathbf{x}} f_{1}^{(\kappa)}(\mathbf{x}, w, p) \quad \text { s.t } \quad(9 e),(10),(23),(31),(32) .
$$

The computational complexity of (33) is [43]

$$
\mathcal{O}\left(\alpha^{2} \beta^{2.5}+\beta^{3.5}\right),
$$

where $\alpha=N M$, which is the number of its decision variables and $\beta=N(3+M)+1$, which is the number of its constraints.

Alternatively, set

$$
\mathbf{x}_{n, m} \equiv 0 \quad \text { whenever } \quad x_{n, m}^{(\kappa)}<\epsilon,
$$

where $\epsilon>0$ is a tolerance for accepting $x_{n, m}=0$ and define

$$
\mathcal{D}_{n}^{(\kappa)} \triangleq\left\{m \in \mathcal{M}: x_{n, m}^{(\kappa)}>\epsilon\right\} .
$$

Applying inequality (2) in Section I for

$$
y=\frac{1}{\mathbf{x}_{n, m}}, x=\frac{p_{m}^{U} g_{n}^{U D}+\sum_{j \in \mathcal{N} \backslash\{n\}} \mathbf{x}_{j, m} p_{j}^{D} g_{j, n}^{D}+w_{m} \sigma_{n}^{2}}{p_{n}^{d} h_{n}^{D}}
$$

and

$$
\bar{y}=\frac{1}{x_{n, m}^{(\kappa)}}, \bar{x}=\frac{p_{m}^{U} g_{n}^{U D}+\sum_{j \in \mathcal{N} \backslash\{n\}} x_{j, m}^{(\kappa)} p_{j}^{D} g_{j, n}^{D}+w_{m} \sigma_{n}^{2}}{p_{n}^{d} h_{n}^{D}}
$$

we obtain

$$
\begin{aligned}
r_{n}^{D}(\mathbf{x}, w, p) \geq & \sum_{m \in \mathcal{D}_{n}^{(\kappa)}}\left[\tilde{\alpha}_{n, m}^{(\kappa)}+\tilde{\beta}_{n, m}^{(\kappa)}\right. \\
& \times \frac{\sum_{j \in \mathcal{N} \backslash\{n\}}\left(x_{j, m}^{(\kappa)}-\mathbf{x}_{j, m}\right) p_{j}^{D} g_{j, n}^{D}}{p_{m}^{U} g_{n}^{U D}+\sum_{j \in \mathcal{N} \backslash\{n\}} x_{j, m}^{(\kappa)} p_{j}^{D} g_{j, n}^{D}+w_{m} \sigma_{n}^{2}}
\end{aligned}
$$




$$
\begin{aligned}
\text { RHS of (27) } & =\sum_{m=1}^{M} \chi_{n, m}^{(\kappa)} \mathbf{x}_{n, m}-\sum_{m=1}^{M} \delta_{n, m}^{(\kappa)} \mathbf{x}_{n, m} \sum_{j \in \mathcal{N} \backslash\{n\}} \mathbf{x}_{j, m} p_{j}^{D} g_{j, n}^{D} \\
& =\sum_{m=1}^{M} \chi_{n, m}^{(\kappa)} \mathbf{x}_{n, m}-\sum_{m=1}^{M} \delta_{n, m}^{(\kappa)}\left[\left(\mathbf{x}_{n, m}+\frac{1}{4} \sum_{j \in \mathcal{N} \backslash\{n\}} \mathbf{x}_{j, m} p_{j}^{D} g_{j, n}^{D}\right)^{2}-\left(\mathbf{x}_{n, m}-\frac{1}{4} \sum_{j \in \mathcal{N} \backslash\{n\}} \mathbf{x}_{j, m} p_{j}^{D} g_{j, n}^{D}\right)^{2}\right] \\
& \geq \sum_{m=1}^{M} \chi_{n, m}^{(\kappa)} \mathbf{x}_{n, m}-\sum_{m=1}^{M} \delta_{n, m}^{(\kappa)}\left(\mathbf{x}_{n, m}+\frac{1}{4} \sum_{j \in \mathcal{N} \backslash\{n\}} \mathbf{x}_{j, m} p_{j}^{D} g_{j, n}^{D}\right)^{2}+\sum_{m=1}^{M} \delta_{n, m}^{(\kappa)}\left(x_{n, m}^{(\kappa)}-\frac{1}{4} \sum_{j \in \mathcal{N} \backslash\{n\}} x_{j, m}^{(\kappa)} p_{j}^{D} g_{j, n}^{D}\right) \\
& \times\left(2 \mathbf{x}_{n, m}-x_{n, m}^{(\kappa)}+\frac{1}{4} \sum_{j \in \mathcal{N} \backslash\{n\}}\left(x_{j, m}^{(\kappa)}-2 \mathbf{x}_{j, m}\right) p_{j}^{D} g_{j, n}^{D}\right) \triangleq r_{n}^{D(\kappa)}(\mathbf{x}, w, p),
\end{aligned}
$$

$$
\begin{gathered}
\left.-\frac{\tilde{\chi}_{n, m}^{(\kappa)}}{\mathbf{x}_{n, m}}\right] \\
\triangleq \tilde{r}_{n}^{D(\kappa)}(\mathbf{x}, w, p),
\end{gathered}
$$

over the trust region ${ }^{2}$

$$
\tilde{r}_{n}^{D(\kappa)}(\mathbf{x}, w, p)>0,
$$

where

$$
\begin{aligned}
\tilde{a}_{n, m}^{(\kappa)} \triangleq & 2 w_{m} x_{n, m}^{(\kappa)} \\
& \times \ln \left(1+\frac{p_{n}^{D} h_{n}^{D}}{p_{m}^{U} g_{n}^{U D}+\sum_{j \in \mathcal{N} \backslash\{n\}} x_{j, m}^{(\kappa)} p_{j}^{D} g_{j, n}^{D}+w_{m} \sigma_{n}^{2}}\right),
\end{aligned}
$$

$$
\tilde{\beta}_{n, m}^{(\kappa)} \triangleq \frac{w_{m} x_{n, m}^{(\kappa)}}{1+\left(p_{m}^{U} g_{n}^{U D}+\sum_{j \in \mathcal{N} \backslash\{n\}} x_{j, m}^{(\kappa)} p_{j}^{D} g_{j, n}^{D}+w_{m} \sigma_{n}^{2}\right) / p_{n}^{D} h_{n}^{D}},
$$

$$
\begin{aligned}
\tilde{\chi}_{n, m}^{(\kappa)} \triangleq & w_{m}\left(x_{n, m}^{(\kappa)}\right)^{2} \\
& \times \ln \left(1+\frac{p_{n}^{D} h_{n}^{D}}{p_{m}^{U} g_{n}^{U D}+\sum_{j=1, j \neq n} x_{j, m}^{(\kappa)} p_{j}^{D} g_{j, n}^{D}+w_{m} \sigma_{n}^{2}}\right) .
\end{aligned}
$$

Instead of (33), we solve the following optimization problem to generate the next iterative point $x^{(\kappa+1)}$ :

$$
\begin{array}{ll} 
& \max _{\mathbf{x}} f_{1}^{(\kappa)}(\mathbf{x}, w, p) \\
\text { s.t. } & (9 e),(10),(23),(38), \\
& \tilde{r}_{n}^{D(\kappa)}(\mathbf{x}, w, p) \geq r_{\text {min }}^{D}, n \in \mathcal{N} .
\end{array}
$$

The computational complexity of (42) is the same at that of (33). We have

$$
f_{\mu}\left(x^{(\kappa)}, w^{(\kappa)}, p^{(\kappa)}\right)=f_{1}^{(\kappa)}\left(x^{(\kappa)}, w^{(\kappa)}, p^{(\kappa)}\right)
$$

${ }^{2}$ The trust region (38) guarantees that $\mathbf{x}_{n, m}>0$ for $m \in \mathcal{D}_{n}^{(\kappa)}$

$$
\begin{aligned}
& <f_{1}^{(\kappa)}\left(x^{(\kappa+1)}, w^{(\kappa)}, p^{(\kappa)}\right) \\
& \leq f_{\mu}\left(x^{(\kappa+1)}, w^{(\kappa)}, p^{(\kappa)}\right),
\end{aligned}
$$

where (43) follows from (26) while (44) is true because $x^{(\kappa+1)}$ and $x^{(\kappa)}$ are the optimal solution and a feasible point for (33), and (45) follows from (24).

\section{B. Resource allocation optimization}

For $\mathbf{x}$ held fixed at $x \triangleq x^{(\kappa+1)}$ we optimize $(\mathbf{w}, \mathbf{p})$ in (14):

$$
\begin{array}{ll} 
& \max _{\mathbf{w}, \mathbf{p}} f_{2}(x, \mathbf{w}, \mathbf{p}) \triangleq \min _{m \in \mathcal{M}} r_{m}^{G}\left(x^{2}, \mathbf{w}, \mathbf{p}\right) \\
\text { s.t } & (9 b)-(9 c),(9 f)-(9 g), \\
& r_{n}^{D}(x, \mathbf{w}, \mathbf{p}) \geq r_{\text {min }}^{D}, n \in \mathcal{N} .
\end{array}
$$

It can be seen from the definitions (7) and (8) that functions $r_{m}^{G}\left(x^{2}, \mathbf{w}, \mathbf{p}\right)$ and $r_{n}^{D}(x, \mathbf{w}, \mathbf{p})$ are of complex structure. We will see right now that the inequalities proved in Section I help to unravel their complexity.

The first step is to apply inequality (4) in Section I for

$$
\begin{aligned}
x=1 / \mathbf{p}_{m}^{U} h_{m}^{U G}, y & =\sum_{n=1}^{N} x_{n, m} \mathbf{p}_{n}^{D} g_{n, m}^{D G}+\mathbf{w}_{m} \sigma_{m}^{2}, \\
t & =1 / \mathbf{w}_{m},
\end{aligned}
$$

and

$$
\begin{aligned}
\bar{x}=1 / p_{m}^{U(\kappa)} h_{m}^{U G}, \bar{y} & =\sum_{n=1}^{N} x_{n, m} p_{n}^{D(\kappa)} g_{n, m}^{D G}+w_{m}^{(\kappa)} \sigma_{m}^{2}, \\
\bar{t} & =1 / w_{m}^{(\kappa)},
\end{aligned}
$$

to obtain

$$
\begin{array}{r}
r_{m}^{G}\left(x^{2}, \mathbf{w}, \mathbf{p}\right) \geq \\
a_{m}^{(\kappa)}+b_{m}^{(\kappa)}\left(1-\frac{p_{m}^{U(\kappa)}}{\mathbf{p}_{m}^{U}}+\right. \\
\left.\frac{\sum_{n=1}^{N}\left(x_{n, m}\right)^{2}\left(p_{n}^{D(\kappa)}-\mathbf{p}_{n}^{D}\right) g_{n, m}^{D G}+\left(w_{m}^{(\kappa)}-\mathbf{w}_{m}\right) \sigma_{m}^{2}}{\sum_{n=1}^{N}\left(x_{n, m}\right)^{2} p_{n}^{D(\kappa)} g_{n, m}^{D G}+w_{m}^{(\kappa)} \sigma_{m}^{2}}\right)
\end{array}
$$




$$
\begin{array}{r}
-\frac{c_{m}^{(\kappa)}}{\mathbf{w}_{m}} \triangleq \\
r_{m}^{G(\kappa)}\left(x^{2}, \mathbf{w}, \mathbf{p}\right),
\end{array}
$$

where

$$
\begin{aligned}
& a_{m}^{(\kappa)} \triangleq 2 w_{m}^{(\kappa)} \ln \left(1+\frac{p_{m}^{U(\kappa)} h_{m}^{U G}}{\sum_{n=1}^{N}\left(x_{n, m}\right)^{2} p_{n}^{D(\kappa)} g_{n, m}^{D G}+w_{m}^{(\kappa)} \sigma_{m}^{2}}\right), \\
& b_{m}^{(\kappa)} \triangleq \frac{w_{m}^{(\kappa)} p_{m}^{U(\kappa)} h_{m}^{U G}}{p_{m}^{U(\kappa)} h_{m}^{U G}+\sum_{n=1}^{N}\left(x_{n, m}\right)^{2} p_{n}^{D(\kappa)} g_{n, m}^{D G}+w_{m}^{(\kappa)} \sigma_{m}^{2}}, \\
& c_{m}^{(\kappa)} \triangleq\left(w_{m}^{(\kappa)}\right)^{2} \ln \left(1+\frac{p_{m}^{U(\kappa)} h_{m}^{U G}}{\sum_{n=1}^{N}\left(x_{n, m}\right)^{2} p_{n}^{D(\kappa)} g_{n, m}^{D G}+w_{m}^{(\kappa)} \sigma_{m}^{2}}\right),
\end{aligned}
$$

which make $r_{m}^{G(\kappa)}\left(x^{2}, \mathbf{w}, \mathbf{p}\right)$ a simple concave function. As a result, the objective function $f_{2}(x, \mathbf{w}, \mathbf{p})$ of minimum throughput in (46a) is lower bounded by a concave function as follows

$$
\begin{aligned}
f_{2}(x, \mathbf{w}, \mathbf{p}) & \geq f_{2}^{(\kappa)}(x, \mathbf{w}, \mathbf{p}) \\
& \triangleq \min _{m \in \mathcal{M}} r_{m}^{G(\kappa)}\left(x^{2}, \mathbf{w}, \mathbf{p}\right)
\end{aligned}
$$

Again function $f_{2}^{(\kappa)}(x, \mathbf{w}, \mathbf{p})$ is concave as minimum of concave functions [40], which also matches with $f_{2}(x, \mathbf{w}, \mathbf{p})$ at $\left(w^{(\kappa)}, p^{(\kappa)}\right)$ :

$$
f_{2}\left(x, w^{(\kappa)}, p^{(\kappa)}\right)=f_{2}^{(\kappa)}\left(x, w^{(\kappa)}, p^{(\kappa)}\right) .
$$

The second step is to provide an inner approximation for the nonconvex constraint (46b). Applying inequality (4) in Section I again for

$$
\begin{aligned}
x=1 / \mathbf{p}_{n}^{D} h_{n}^{D}, y & =\mathbf{p}_{m}^{U} g_{n}^{U D}+\sum_{j \in \mathcal{N} \backslash\{n\}} x_{j, m} \mathbf{p}_{j}^{D} g_{j, n}^{D}+\mathbf{w}_{m} \sigma_{n}^{2}, \\
t & =1 / \mathbf{w}_{m},
\end{aligned}
$$

and

$$
\begin{aligned}
\bar{x} & =1 / p_{n}^{D(\kappa)} h_{n}^{D}, \\
\bar{y} & =p_{m}^{U(\kappa)} g_{n}^{U D}+\sum_{j \in \mathcal{N} \backslash\{n\}} x_{j, m} p_{j}^{D(\kappa)} g_{j, n}^{D}+w_{m}^{(\kappa)} \sigma_{n}^{2}, \\
\bar{t} & =1 / w_{m}^{(\kappa)}
\end{aligned}
$$

yields

$$
\begin{array}{r}
r_{n}^{D}(x, \mathbf{w}, \mathbf{p}) \\
\sum_{m \in \mathcal{D}_{n}^{(\kappa)}}\left[\alpha_{n, m}^{(\kappa)}+\beta_{n, m}^{(\kappa)}\left(2-\frac{p_{n}^{D(\kappa)}}{\mathbf{p}_{n}^{D}}-\right.\right. \\
\left.\mathbf{p}_{m}^{U} g_{n}^{U D}+\sum_{j \in \mathcal{N} \backslash\{n\}} x_{j, m} \mathbf{p}_{j}^{D} g_{j, n}^{D}+\mathbf{w}_{m} \sigma_{n}^{2}\right) \\
\sum_{m}^{U(\kappa)} g_{n}^{U D}+\sum_{j \in \mathcal{N} \backslash\{n\}} x_{j, m} p_{j}^{D(\kappa)} g_{j, n}^{D}+w_{m}^{(\kappa)} \sigma_{n}^{2} \\
\left.-\frac{\chi_{n, m}^{(\kappa)}}{\mathbf{w} m}\right]
\end{array}
$$

where

$$
\begin{aligned}
0 & <\alpha_{n, m}^{(\kappa)}=2 x_{n, m} w_{m}^{(\kappa)} \\
& \times \ln \left(1+\frac{p_{n}^{D(\kappa)} h_{n}^{D}}{p_{m}^{U(\kappa)} g_{n}^{U D}+\sum_{j \in \mathcal{N} \backslash\{n\}} x_{j, m} p_{j}^{D(\kappa)} g_{j, n}^{D}+w_{m}^{(\kappa)} \sigma_{n}^{2}}\right), \\
0 & <\beta_{n, m}^{(\kappa)}=x_{n, m} w_{m}^{(\kappa)} \\
& \times \frac{p_{n}^{D(\kappa)} h_{n}^{D}+p_{m}^{U(\kappa)} g_{n}^{U D}+\sum_{j \in \mathcal{N} \backslash\{n\}}^{D(\kappa)} h_{n}^{D}}{p_{j, m} p_{j}^{D(\kappa)} g_{j, n}^{D}+w_{m}^{(\kappa)} \sigma_{n}^{2}}, \\
0 & <\chi_{n, m}^{(\kappa)}=x_{n, m}\left(w_{m}^{(\kappa)}\right)^{2} \\
& \times \ln \left(1+\frac{(56)}{p_{m}^{U(\kappa)} g_{n}^{U D}+\sum_{j \in \mathcal{N} \backslash\{n\}} x_{j, m} p_{j}^{D(\kappa)} g_{j, n}^{D}+w_{m}^{(\kappa)} \sigma_{n}^{2}}\right),
\end{aligned}
$$

which make $r_{n}^{D(\kappa)}(x, \mathbf{w}, \mathbf{p})$ a concave function of $(\mathbf{w}, \mathbf{p})$. The nonconvex constraint (46b) is now innerly approximated by the convex constraint

$$
r_{n}^{D(\kappa)}(x, \mathbf{w}, \mathbf{p}) \geq r_{\text {min }}^{D}, n \in \mathcal{N} .
$$

We solve the following inner approximation problem of (17) to generate the next iterative point $\left(w^{(\kappa+1)}, p^{(\kappa+1)}\right)$ :

$$
\max _{\mathbf{w}, \mathbf{p}} f_{2}^{(\kappa)}(x, \mathbf{w}, \mathbf{p}) \quad \text { s.t. } \quad(9 b)-(9 c),(9 f)-(9 g),(58) .
$$

The computational complexity of (59) is (34) for $\alpha=2 M+$ $N$ and $\beta=2 N+M+2$.

We have

$$
\begin{aligned}
f_{2}\left(x^{(\kappa+1)}, w^{(\kappa)}, p^{(\kappa)}\right) & = \\
f_{2}^{(\kappa)}\left(x^{(\kappa+1)}, w^{(\kappa)}, p^{(\kappa)}\right) & < \\
f_{2}^{(\kappa)}\left(x^{(\kappa+1)}, w^{(\kappa+1)}, p^{(\kappa+1)}\right) & \leq \\
f_{2}\left(x^{(\kappa+1)}, w^{(\kappa+1)}, p^{(\kappa+1)}\right), &
\end{aligned}
$$

where (60) follows from (53) while (61) is true because $\left(w^{(\kappa+1)}, p^{(\kappa+1)}\right)$ and $\left(w^{(\kappa)}, p^{(\kappa)}\right)$ are the optimal solution and a feasible point for (59), and (62) follows from (51), which results in

$$
\begin{array}{r}
f_{\mu}\left(x^{(\kappa+1)}, w^{(\kappa)}, p^{(\kappa)}\right)= \\
f_{2}\left(x^{(\kappa+1)}, w^{(\kappa)}, p^{(\kappa)}\right)+\mu \widetilde{g}\left(x^{(\kappa+1)}\right)< \\
f_{\mu}^{2}\left(x^{(\kappa+1)}, w^{(\kappa+1)}, p^{(\kappa+1)}\right)+\mu \widetilde{g}\left(x^{(\kappa+1)}\right)= \\
f_{\mu}\left(x^{(\kappa+1)}, w^{(\kappa+1)}, p^{(\kappa+1)}\right) .
\end{array}
$$

\section{Initialization}

It follows from (59) that initialized by a feasible point $\left(w^{0)}, p^{(0)}\right)$ for the convex constraints $(9 \mathrm{~b})-(9 \mathrm{c})$ and $(9 \mathrm{f})-(9 \mathrm{~g})$, we iterate

$$
\max _{\mathbf{w}, \mathbf{p}} \min \left\{\min _{m \in \mathcal{M}} \frac{r_{m}^{G(\kappa)}\left(\left(x^{(0)}\right)^{2}, \mathbf{w}, \mathbf{p}\right)}{r_{\min }^{U}},\right.
$$


$\overline{\text { Algorithm } 1 \text { Alternating descent maximin GT throughput }}$ optimization algorithm

Initialization Take any feasible point $x^{(0)}$ for (9e), (10). Using iterations (64) to find a feasible point $\left(x^{(0)}, w^{(\kappa)}, p^{(\kappa)}\right)$ for (14). Calculate $\mu$ according to (16). Set $\kappa=0$.

repeat

Solve (33) or (42) for $(w, p)=\left(w^{(\kappa)}, p^{(\kappa)}\right)$ to generate $x^{(\kappa+1)}$.

Solve (59) for $x=x^{(\kappa+1)}$ to generate $\left(w^{(\kappa+1)}, p^{(\kappa+1)}\right)$.

until convergence
Reset $\kappa \leftarrow \kappa+1$.

s.t. $(9 f)-(9 g)$,

$\mathbf{w}_{m} \geq 0, m \in \mathcal{M} ; \mathbf{w}_{D} \geq 0$,

$\sum_{m=1}^{M} \mathbf{w}_{m}+\mathbf{w}_{D}=\mathcal{B}$,

$\tilde{r}_{n}^{D}\left(\mathbf{w}_{D}, \mathbf{p}^{U}\right) \geq \tilde{r}_{\text {min }}^{D}, n \in \mathcal{N}$,

Let $\left(w^{(\kappa)}, w_{D}^{(\kappa)}, p^{(\kappa)}\right)$ be a feasible point for (68) found from the $\kappa$ th iteration. Applying (4) in Section I for

$$
x=1 / \mathbf{p}_{m}^{G} h_{m}^{U G}, y=\mathbf{w}_{m} \sigma_{m}^{2}, t=1 / \mathbf{w}_{m}
$$

and

$$
\bar{x}=p_{m}^{(G(\kappa))}, \bar{y}=w_{m}^{(\kappa)} \sigma^{2}, \bar{t}=1 / w_{m}^{(\kappa)}
$$

yields

$$
\begin{aligned}
\tilde{r}_{m}^{G}\left(\mathbf{w}_{m}, \mathbf{p}_{m}^{U}\right) & \geq a_{m}^{(\kappa)}+b_{m}^{(\kappa)}\left(2-\frac{p_{m}^{U(\kappa)}}{\mathbf{p}_{m}^{U}}-\frac{\mathbf{w}_{m}}{w_{m}^{(\kappa)}}\right)-\frac{c_{m}^{(\kappa)}}{\mathbf{w}_{m}} \\
& \triangleq \tilde{r}_{m}^{G(\kappa)}\left(\mathbf{w}_{m}, \mathbf{p}_{m}^{U}\right),
\end{aligned}
$$

than or equal to 1 , making $\left(x^{0}, w^{(\kappa)}, p^{(\kappa)}\right)$ feasible for (14) and thus qualifies as an initial point for the above proposed alternating decent process.

\section{Alternating descent algorithm}

Algorithm 1 summarizes our proposed alternating descent procedure. Inequalities (45) and (63) yield

$$
f_{\mu}\left(x^{(\kappa)}, w^{(\kappa)}, p^{(\kappa)}\right)<f_{\mu}\left(x^{(\kappa+1)}, w^{(\kappa+1)}, p^{(\kappa+1)}\right) .
$$

As such $\left\{x^{(\kappa)}, w^{(\kappa)}, p^{(\kappa)}\right\}$ is a sequence of improved feasible points for (14), which converges to its feasible point $(\bar{x}, \bar{w}, \bar{p})$ such that $\bar{x}$ is a Karush-Kuh-Tucker (KKT) point of (17) for $(w, p)=(\bar{w}, \bar{p})$ while $(\bar{w}, \bar{p})$ is a KKT point of (46) for $x=\bar{x}$ [42].

\section{NON-COGNITIVE SCENARIO}

It can be seen from (8) that the UAV interference term $\mathbf{p}_{m}^{U} g_{n}^{U D}$, which is strong compared to D2D' interference term $\sum_{j \in \mathcal{N} \backslash\{n\}} \mathbf{x}_{j, m} \mathbf{p}_{j}^{D} g_{j, n}^{D}$ because the path gain $g_{n}^{U D}$ of LoS is much larger than path gains $g_{j, n}^{D}$ of NLoS, is a major factor limiting D2D throughput. As such, the UAV interference is enhanced to guarantee the QoS requirement (9h) for the D2D users. We now consider a non-cognitive network, which dedicates a partial bandwidth resource $\mathbf{w}_{D}$ with $\mathbf{w}_{D}<\mathcal{B}$ to reject the UAV interference completely. The throughput at the $m$-th GT and $n$-th D2D pair are now

$$
\tilde{r}_{m}^{G}\left(\mathbf{w}_{m}, \mathbf{p}_{m}^{U}\right)=\mathbf{w}_{m} \ln \left(1+\frac{\mathbf{p}_{m}^{U} h_{m}^{U G}}{\mathbf{w}_{m} \sigma_{m}^{2}}\right),
$$

and

$$
\tilde{r}_{n}^{D}\left(\mathbf{w}_{D}, \mathbf{p}^{D}\right)=\mathbf{w}_{D} \ln \left(1+\frac{\mathbf{p}_{n}^{D} h_{n}^{D}}{\sum_{j \in \mathcal{N} \backslash\{n\}} \mathbf{p}_{j}^{D} g_{j, n}^{D}+\mathbf{w}_{D} \sigma_{n}^{2}}\right),
$$

which is free from the UAV interference. The GT throughput maximin problem for non-cognitive networks can be formulated as

$$
\max _{\mathbf{w}, \mathbf{w}_{D}, \mathbf{p}} \tilde{f}\left(\mathbf{w}, \mathbf{p}^{U}\right) \triangleq \min _{m \in \mathcal{M}} \tilde{r}_{m}^{G}\left(\mathbf{w}_{m}, \mathbf{p}_{m}^{U}\right)
$$

$$
\begin{aligned}
a_{m}^{(\kappa)} & \triangleq 2 w_{m}^{(\kappa)} \ln \left(1+\frac{p_{m}^{U(\kappa)} h_{m}^{U G}}{w_{m}^{(\kappa)} \sigma_{m}^{2}}\right), \\
b_{m}^{(\kappa)} & \triangleq \frac{w_{m}^{(\kappa)} p_{m}^{U(\kappa)} h_{m}^{U G}}{p_{m}^{U(\kappa)} h_{m}^{U G}+w_{m}^{(\kappa)} \sigma_{m}^{2}}, \\
c_{m}^{(\kappa)} & \triangleq\left(w_{m}^{(\kappa)}\right)^{2} \ln \left(1+\frac{p_{m}^{U(\kappa)} h_{m}^{U G}}{w_{m}^{(\kappa)} \sigma_{m}^{2}}\right) .
\end{aligned}
$$

Applying inequality (4) in Section I again for

$$
x=1 / \mathbf{p}_{n}^{D} h_{n}^{D}, y=\sum_{j \in \mathcal{N} \backslash\{n\}} \mathbf{p}_{j}^{D} g_{j, n}^{D}+\mathbf{w}_{D} \sigma_{n}^{2}, t=1 / \mathbf{w}_{D}
$$

and

$$
\bar{x}=1 / p_{n}^{D(\kappa)}, \bar{y}=\sum_{j \in \mathcal{N} \backslash\{n\}} p_{j}^{D(\kappa)} g_{j, n}^{D}+w_{D}^{(\kappa)} \sigma_{n}^{2}, t=1 / w_{D}^{(\kappa)}
$$

we obtain

$$
\begin{array}{r}
\tilde{r}_{n}^{D}\left(\mathbf{w}_{D}, \mathbf{p}^{D}\right) \geq \\
\alpha_{n}^{(\kappa)}+\beta_{n}^{(\kappa)}\left(1-\frac{p_{n}^{D(\kappa)}}{\mathbf{p}_{n}^{D}}\right. \\
\left.+\frac{\sum_{j \in \mathcal{N} \backslash\{n\}}\left(p_{j}^{D(\kappa)}-\mathbf{p}_{j}^{D}\right) g_{j, n}^{D}+\left(w_{D}^{(\kappa)}-\mathbf{w}_{D}\right) \sigma_{n}^{2}}{\sum_{j \in \mathcal{N} \backslash\{n\}} p_{j}^{D(\kappa)} g_{j, n}^{D}+w_{D}^{(\kappa)} \sigma_{n}^{2}}\right)-\frac{\chi_{n}^{(\kappa)}}{\mathbf{w}_{D}} \triangleq \\
\tilde{r}_{n}^{D(\kappa)}\left(\mathbf{w}_{D}, \mathbf{p}^{D}\right),
\end{array}
$$

where

$$
\begin{aligned}
& \alpha_{n}^{(\kappa)} \triangleq 2 w_{D}^{(\kappa)} \ln \left(1+\frac{p_{n}^{D(\kappa)} h_{n}^{D}}{\sum_{j \in \mathcal{N} \backslash\{n\}} p_{j}^{D(\kappa)} g_{j, n}^{D}+w_{D}^{(\kappa)} \sigma_{n}^{2}}\right), \\
& \beta_{n}^{(\kappa)} \triangleq \frac{w_{D}^{(\kappa)} p_{n}^{D(\kappa)} h_{n}^{D}}{p_{n}^{D(\kappa)} h_{n}^{D}+\sum_{j \in \mathcal{N} \backslash\{n\}} p_{j}^{D(\kappa)} g_{j, n}^{D}+w_{D}^{(\kappa)} \sigma_{n}^{2}},
\end{aligned}
$$


$\overline{\text { Algorithm } 2 \text { Path-following GT throughput optimization al- }}$ gorithm for non-cognitive networks

Initialization Taking any feasible point $w^{(0)}, p^{(0)}$ for (9f)$(9 \mathrm{~g})$ and $(68 \mathrm{~b})-(68 \mathrm{c})$. Using iterations (70) to find a feasible point $\left(w^{(\kappa)}, p^{(\kappa)}\right)$ for (68). Set $\kappa=0$.

repeat

Solve $(69 \mathrm{~b})$ to generate $\left(w^{(\kappa+1)}, p^{(\kappa+1)}\right)$.

Reset $\kappa \leftarrow \kappa+1$.

until convergence

$$
\chi_{n}^{(\kappa)} \triangleq\left(w_{D}^{(\kappa)}\right)^{2} \ln \left(1+\frac{p_{n}^{D(\kappa)} h_{n}^{D}}{\sum_{j \in \mathcal{N} \backslash\{n\}} p_{j}^{D(\kappa)} g_{j, n}^{D}+w_{D}^{(\kappa)} \sigma_{n}^{2}}\right) .
$$

We solve the following inner approximation problem of (68) to generate the next iterative point $\left(w^{(\kappa+1)}, p^{(\kappa+1)}\right)$ :

$$
\begin{array}{ll} 
& \max _{\mathbf{w}, \mathbf{w}_{D}, \mathbf{p}} f_{3}^{(\kappa)}(\mathbf{w}, \mathbf{p}) \triangleq \min _{m \in \mathcal{M}} \tilde{r}_{m}^{G(\kappa)}\left(\mathbf{w}_{m}, \mathbf{p}_{m}^{U}\right) \\
\text { s.t. } & (9 f)-(9 g),(68 b)-(68 c), \\
& \tilde{r}_{n}^{D(\kappa)}\left(\mathbf{w}_{D}, \mathbf{p}^{D}\right) \geq \tilde{r}_{\min }^{D}, n \in \mathcal{N},
\end{array}
$$

where the objective function $f_{3}^{(\kappa)}$ in (69a) is concave lower bounding function for the nonconcave objective function in (68a) and the convex constraint (69b) (because function $\tilde{r}_{n}^{D(\kappa)}$ is concave) is an inner approximation for the nonconvex constraint (68d). Functions $f_{3}^{(\kappa)}$ and $\tilde{r}_{n}^{D(\kappa)}$ respectively match with functions $f_{3}$ and $\tilde{r}_{n}^{D}$ at $\left(w^{(\kappa)}, w_{D}^{(\kappa)}, p^{(\kappa)}\right)$.

Initialization. Initialized by a feasible point $\left(w^{0)}, p^{(0)}\right)$ for the convex constraints $(9 \mathrm{f})-(9 \mathrm{~g})$ and $(68 \mathrm{~b})-(68 \mathrm{c})$, we iterate

$$
\begin{array}{r}
\max _{\mathbf{w}, \mathbf{p}} \min \left\{\min _{m \in \mathcal{M}} \frac{\tilde{r}_{m}^{G(\kappa)}\left(\mathbf{w}_{m}, \mathbf{p}\right)}{\tilde{r}_{\min }^{U}}, \min _{n \in \mathcal{N}} \frac{\tilde{r}_{n}^{D(\kappa)}\left(\mathbf{w}_{D}, \mathbf{p}\right)}{\tilde{r}_{\min }^{D}}\right\} \\
\text { s.t. }(9 f)-(9 g),(68 b)-(68 c)
\end{array}
$$

for $\kappa=0, \ldots$, till the objective value of (70) reaches more than or equal to 1 , making $\left(w^{(\kappa)}, p^{(\kappa)}\right)$ feasible and thus qualifies as an initial point for (68).

By Algorithm 2 we propose a path-following algorithm for (68) as it iterates improved feasible points for (68) in making them converge to an optimal solution of (68).

\section{Simulation Results}

\section{A. System setup}

There are $M=5$ GTs and $N=20$ D2D pairs, which are uniformly distributed in the circle area with radius $R=500$ $\mathrm{m}$, as shown in Fig. 2. For each D2D pair, the communication distance is close enough to satisfy the distance requirement of D2D communication, i.e., $R_{D}<30 \mathrm{~m}$. The error tolerance of the algorithms is set as $\epsilon=1 \mathrm{e}-4$. The NLoS channels $h_{n}^{D}$ and $g_{j, n}^{D}$ of D2D communication and the interference link $g_{n, m}^{D G}$ between the D2D transmitters and the GTs in cognitive UAVenabled networks are modeled as $\omega_{0} d^{-\alpha_{N L o S}} \delta$, where $d$ is the distance (in meters) and $\alpha_{N L o s} \geq 2$ is the NLoS path-loss exponent while $\delta$ is the Rayleigh fading coefficient, which follows the exponential distribution with unit mean. The values of useful parameters are summarized by Table I [13], [34].

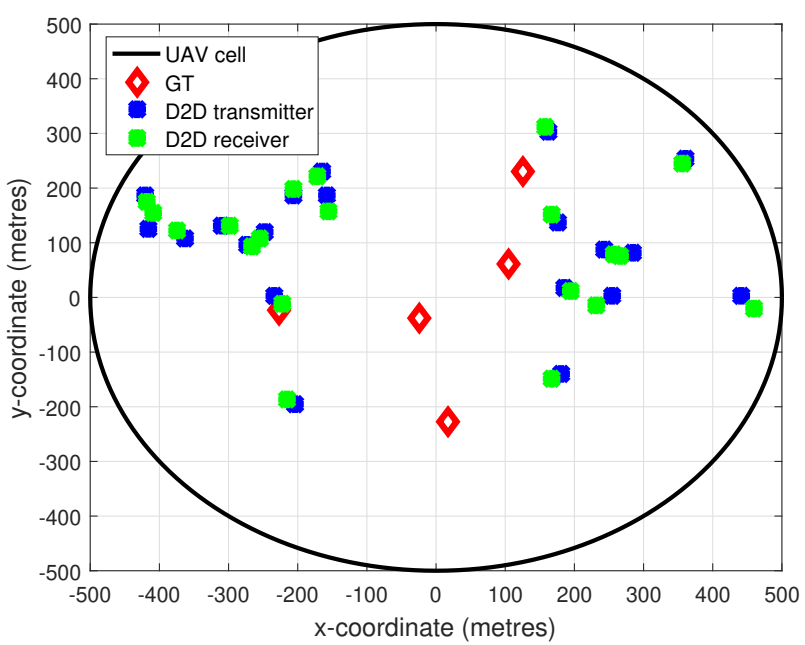

Fig. 2. Scenario with $M=5$ GTs and $N=20$ D2D pairs are uniformly distributed.

TABLE I

PARAMETER SetTings

\begin{tabular}{l||l}
\hline Parameter & Value \\
\hline UAV altitude & $100 \mathrm{~m}$ \\
Carrier frequency $\left(f_{c}\right) / \operatorname{Bandwidth}(\mathcal{B})$ & $2 \mathrm{GHz} / 10 \mathrm{MHz}$ \\
Path-loss exponent $\left(\alpha_{N L o S}\right)$ & 3 \\
Noise power density & $-169 \mathrm{dBm} / \mathrm{Hz}$ \\
The maximum transmit power of D2D & $0.1 \mathrm{~W}$ \\
\hline
\end{tabular}

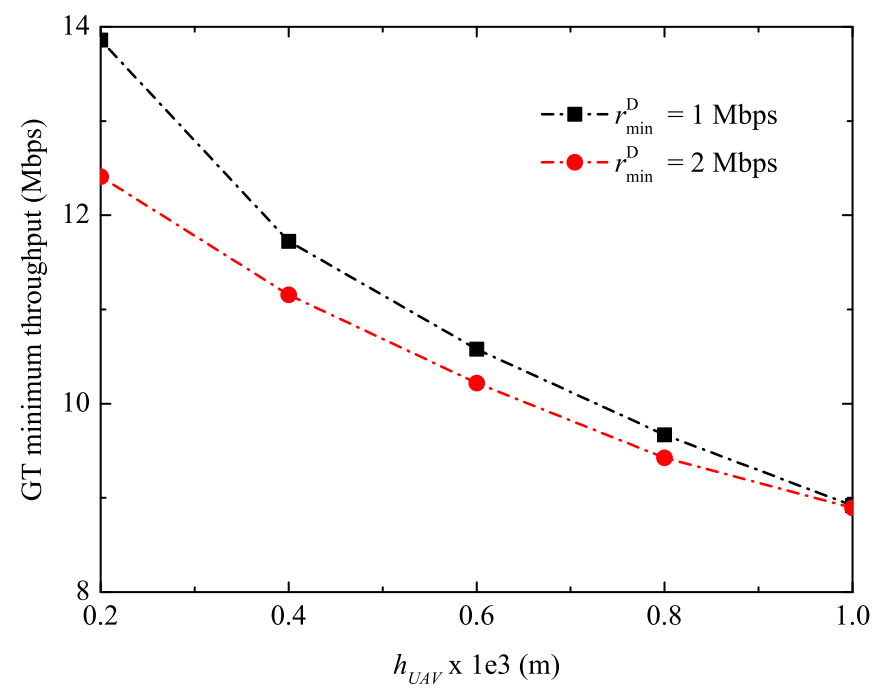

Fig. 3. GT minimum throughput versus $h_{U A V}$ with $P_{\max }^{U}=0.1 \mathrm{~W}$.

\section{B. Cognitive network performance}

Fig. 3 plots the GTs' throughput performance under different UAV altitudes $h_{U A V}$ with the maximum transmit power $P_{\max }^{U}$ fixed at $0.1 \mathrm{~W}$. LoS channel gains are stronger under UAV lower attitudes, which lead to higher GT throughput as far as D2D QoS in terms of $r_{\min }^{D}$ is still preserved. This means that the optimal UAV altitude is the lowest allowable attitude, which is also optimal with D2D users ignored. Moreover, it is well known [38] that the UAV consumes the lowest energy 


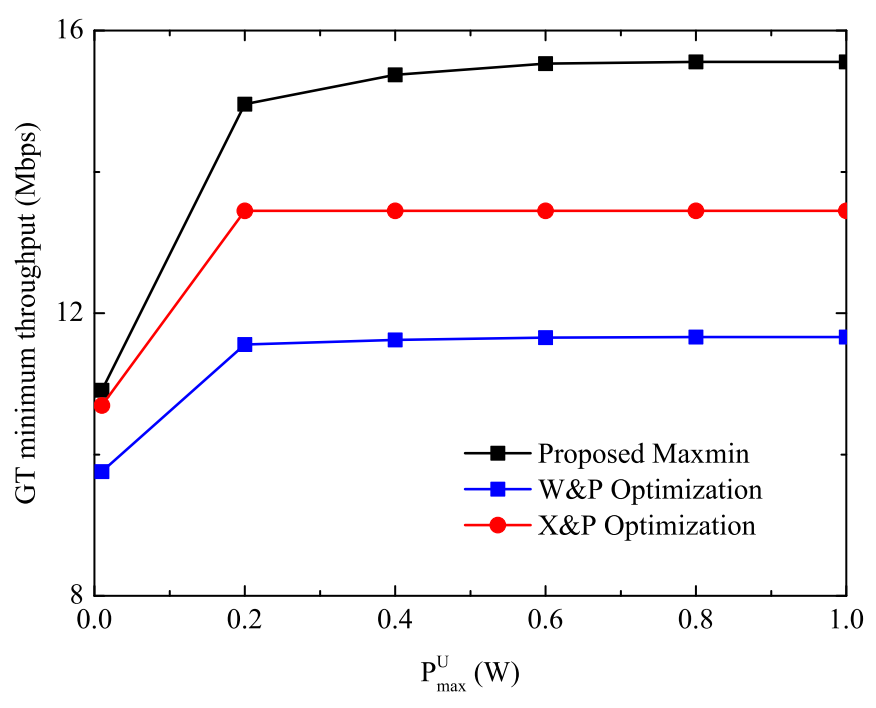

Fig. 4. GT minimum throughput versus $P_{\max }^{U}$ with $r_{\min }^{D}=2 \mathrm{Mbps}$.

when it hovers and keeps its position stationary, so there is no reason for the UAV to move around this position.

The GT minimum throughput versus $P_{\max }^{U}$ is plotted in Fig. 4 with the fixed $r_{\min }^{D}=2 \mathrm{Mbps}$. It increases sensibly in the range of $0<P_{\max }^{U} \leq 0.2 \mathrm{~W}$ but then increases slightly only for $0.2 \mathrm{~W}<P_{\max }^{U}<0.6 \mathrm{~W}$, and becomes saturated for $P_{\max }^{U}>0.6 \mathrm{~W}$. Fig. 5, which plots the UAV's transmit power $\sum_{m=1}^{M} p_{m}^{U}$ versus $P_{\max }^{U}$, particularly clarifies this outcome: the UAV cannot use more than $0.6 \mathrm{~W}$ for transmission to control its interference to the underlaid D2D users in supporting the required $\mathrm{QoS}$ for them.

Fig. 4 also shows the advantage of the proposed joint optimization over the "W\&P Optimization" and "X\&P Optimization". In the "W\&P Optimization", the D2D assignment is random such that the constraints $(9 \mathrm{~d})-(9 \mathrm{e})$ are satisfied and then the optimization problem (59) in (w, p) is solved until its convergence. In the "X\&P Optimization", only D2D assignment and power allocation are optimized while $\mathbf{w}_{m} \equiv 1 / M$, i.e, GTs are allocated by the equal bandwidths. It can be seen that the proposed joint optimization significantly outperforms others in terms of GT throughput.

\section{Cognitive performance vs non-cognitive performance}

This subsection compares the performance by the cognitive communication network described in Section II and its noncognitive counterpart described in Section IV, in terms of the minimum GT throughput. Fig. 6 shows that the cognitive network outperforms the non-cognitive one for the practical UAV transmit power range, i.e., $P_{\max }^{U} \leq 0.1 \mathrm{~W}$. The reduction of GT bandwidth in the non-cognitive network cannot be compensated by optimizing the UAV transmit power. The non-cognitive network outperforms the cognitive network only for unpractically high UAV transmit power range such as $P_{\max }^{U} \geq 5 \mathrm{~W}$ because the latter cannot use such high power, which would hurt D2D throughput very much, while the former can certainly do so to enhance the GT throughput as it does not effect on D2D throughput.

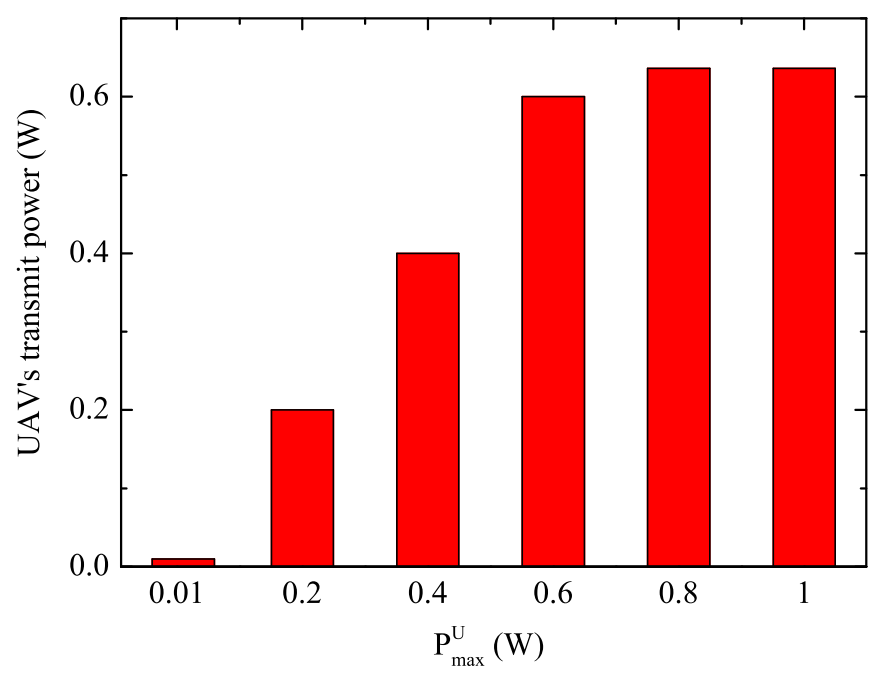

Fig. 5. UAV transmit power.

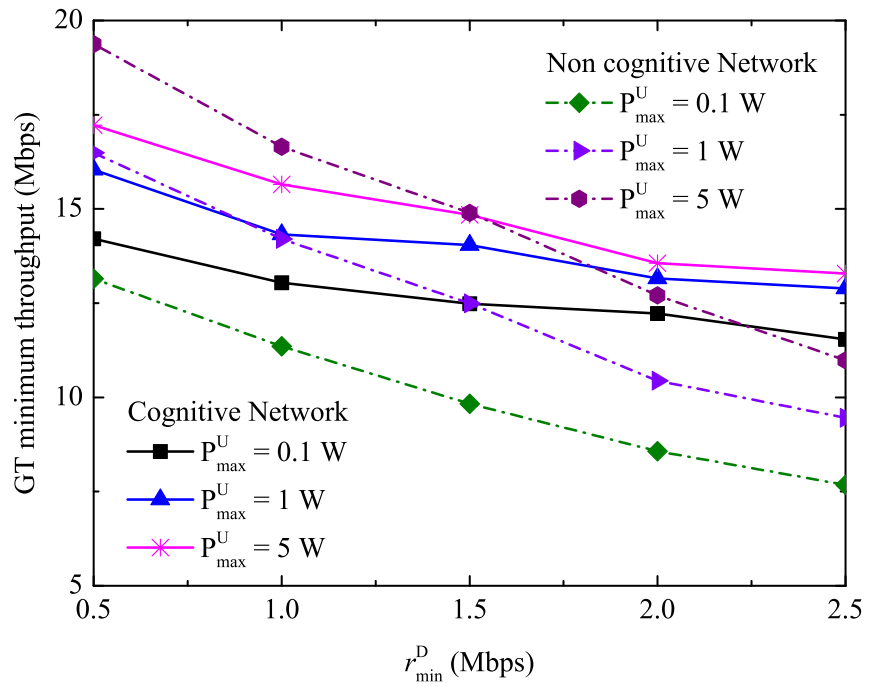

Fig. 6. GT minimum throughput versus $r_{\min }^{D}$.

\section{Algorithms' convergence}

Fig. 7 and Fig. 8 plot the typical convergence of Algorithm 1 and Algorithm 2 for $P_{\max }^{U}=0.1 \mathrm{~W}$ with different $r_{\min }^{D}$ levels, respectively. As can be seen from Fig. 8, Algorithm 2 converges more rapidly than Algorithm 1, taking around 6 iterations for convergence. Indeed, Algorithm 1 needs to deal not only bandwidth and power allocation but also D2D assignment at the same iteration. This results also demonstrate the increasing objective functions at each iteration. In addition, Fig. 7(b) shows the convergence to zero of the penalty function in (13), which results in binary values of the assignment variables.

\section{Conclusions}

This paper has considered a cognitive communication network, which consists of an UAV-enabled communication to serve multiple GT users, and multiple cognitive D2D users. 


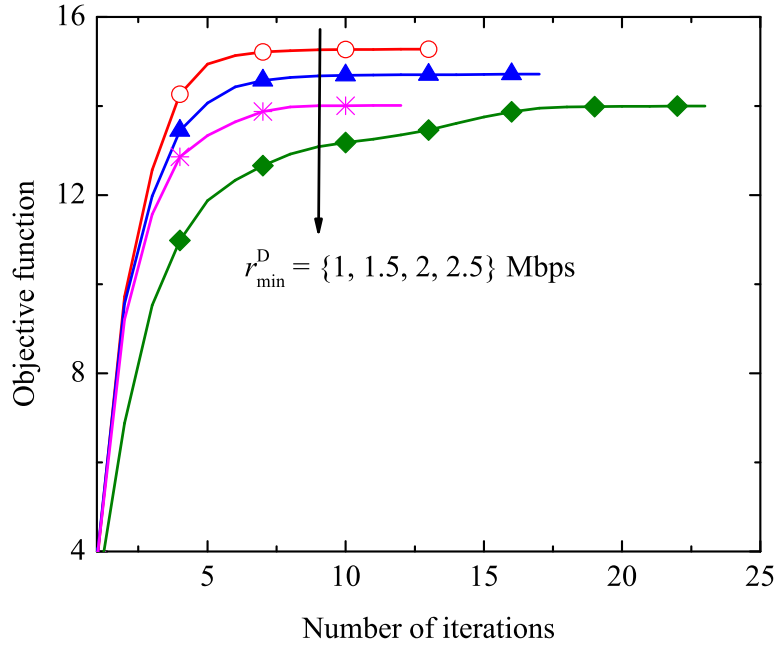

(a) Original objective function.

Fig. 7. Convergence of Algorithm 1 for $P_{\max }^{U}=0.1 \mathrm{~W}$.

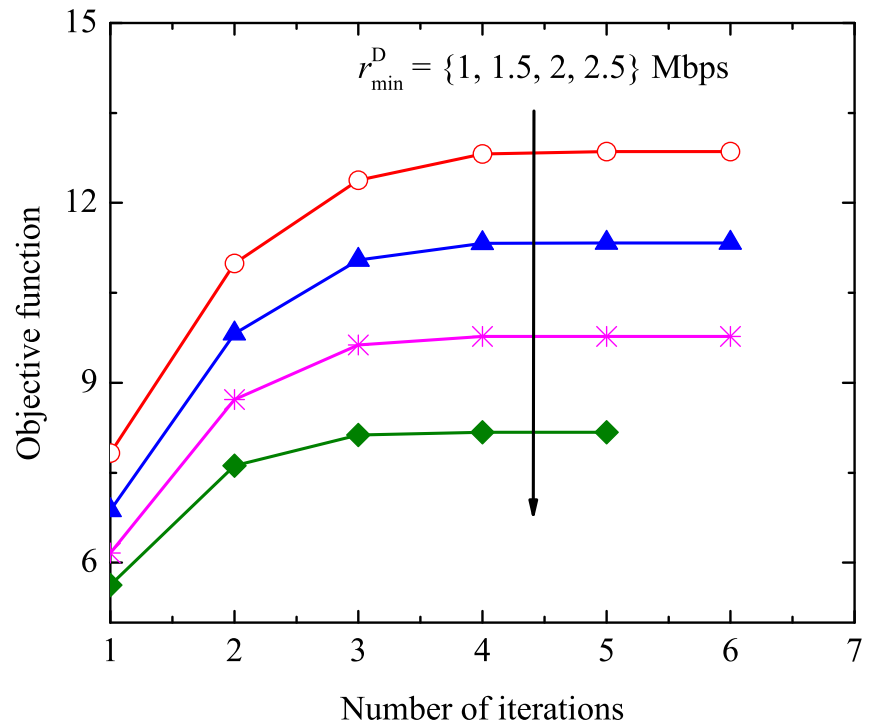

Fig. 8. Convergence of Algorithm 2 for $P_{\max }^{U}=0.1 \mathrm{~W}$.

The joint design of D2D assignment, bandwidth and power allocation problem has been proposed to enhance the GT throughput subject to the QoS of D2D communication. A noncognitive network was also examined to show the advantages of the cognitive exploitation. All the design problems pose difficult optimization problems, for which the paper has developed efficient solvers of low computational complexity. The numerical results have been provided to show their ability in supporting high GT throughput while guaranteeing the required QoS for the D2D users.

Exploiting improper Gaussian signaling to further improve D2D throughput is under current study.

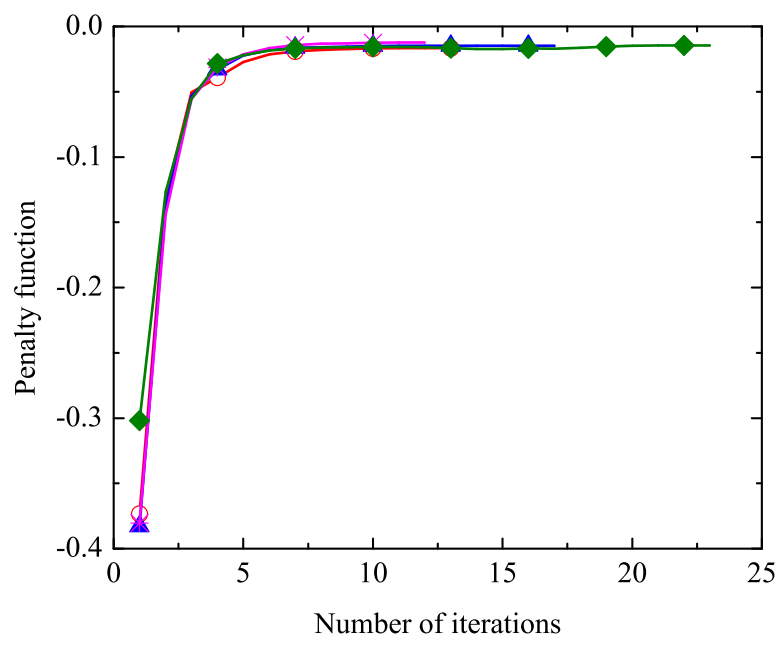

(b) Penalty function.

\section{REFERENCES}

[1] A. Merwaday, A. Tuncer, A. Kumbhar, and I. Guvenc, "Improved throughput coverage in natural disasters: Unmanned aerial base stations for public-safety communications," IEEE Veh. Technol. Mag., vol. 11, no. 4, pp. 53-60, Dec. 2016.

[2] Y. Zeng, R. Zhang, and T. J. Lim, "Wireless communications with unmanned aerial vehicles: opportunities and challenges," IEEE Commun. Mag., vol. 54, no. 5, pp. 36-42, May 2016.

[3] H. Menouar, I. Guvenc, K. Akkaya, A. S. Uluagac, A. Kadri, and A. Tuncer, "UAV-enabled intelligent transportation systems for the smart city: Applications and challenges," IEEE Commun. Mag., vol. 55, no. 3, pp. 22-28, Mar. 2017.

[4] M. Mozaffari, W. Saad, M. Bennis, and M. Debbah, "Mobile unmanned aerial vehicles (UAVs) for energy-efficient internet of things communications," IEEE Trans. Wirel. Commun., vol. 16, no. 11, pp. 7574-7589, Nov. 2017.

[5] GSMA. Mobile-enabled unmanned aircraft. [Online]. Available: https://www.gsma.com/iot/mobile-enabled-unmanned-aircraft/

[6] P. Zhan, K. Yu, and A. L. Swindlehurst, "Wireless relay communications with unmanned aerial vehicles: Performance and optimization," IEEE Trans. Aerosp. Electron. Syst., vol. 47, no. 3, pp. 2068-2085, Jul. 2011.

[7] M. Erdelj, E. Natalizio, K. R. Chowdhury, and I. F. Akyildiz, "Help from the sky: Leveraging UAVs for disaster management," IEEE Pervasive Comput., vol. 16, no. 1, pp. 24-32, Jan. 2017.

[8] M. Mozaffari, W. Saad, M. Bennis, and M. Debbah, "Efficient deployment of multiple unmanned aerial vehicles for optimal wireless coverage," IEEE Commun. Lett., vol. 20, no. 8, pp. 1647-1650, Aug. 2016.

[9] V. V. Chetlur and H. S. Dhillon, "Downlink coverage analysis for a finite 3-D wireless network of unmanned aerial vehicles," IEEE Trans. Commun., vol. 65, no. 10, pp. 4543-4558, Oct. 2017.

[10] Y. Chen, W. Feng, and G. Zheng, "Optimum placement of UAV as relays," IEEE Commun. Lett., vol. 22, no. 2, pp. 248-251, Feb. 2018.

[11] Y. Zeng and R. Zhang, "Energy-efficient UAV communication with trajectory optimization," IEEE Trans. Wirel. Commun., vol. 16, no. 6, pp. 3747-3760, Jun. 2017.

[12] Y. Zeng, X. Xu, and R. Zhang, "Trajectory design for completion time minimization in UAV-enabled multicasting," IEEE Trans. Wirel. Commun., vol. 17, no. 4, pp. 2233-2246, Apr. 2018.

[13] J. Lyu, Y. Zeng, and R. Zhang, "UAV-Aided offloading for cellular hotspot," IEEE Trans. Wirel. Commun., vol. 17, pp. 3988-4001, Jun. 2018.

[14] H. He, S. Zhang, Y. Zeng, and R. Zhang, "Joint altitude and beamwidth optimization for UAV-enabled multiuser communications," IEEE Commun. Lett., vol. 22, no. 2, pp. 344-347, Feb. 2018.

[15] Y. Zeng, R. Zhang, and T. J. Lim, "Throughput maximization for UAV-enabled mobile relaying systems," IEEE Trans. Commun., vol. 64, no. 12, pp. 4983-4996, Dec. 2016. 
[16] H. Ghazzai, M. B. Ghorbel, A. Kadri, M. J. Hossain, and H. Menouar, "Energy-efficient management of unmanned aerial vehicles for underlay cognitive radio systems," IEEE Trans. Green Commun. Netw., vol. 1, no. 4, pp. 434-443, Dec. 2017.

[17] H. Wang, J. Wang, G. Ding, L. Wang, T. A. Tsiftsis, and P. K. Sharma, "Resource allocation for energy harvesting-powered D2D communication underlaying UAV-assisted networks," IEEE Trans. Green Commun. Netw., vol. 2, no. 1, pp. 14-24, Mar. 2018.

[18] M.-N. Nguyen, L. D. Nguyen, T. Q. Duong, and H. D. Tuan, "Realtime optimal resource allocation for embedded UAV communication systems," IEEE Wirel. Commun. Lett., vol. 8, no. 1, pp. 225-228, Jan. 2019.

[19] L. Sboui, H. Ghazzai, Z. Rezki, and M.-S. Alouini, "Energy-efficient power allocation for UAV cognitive radio systems," in Proc. IEEE 86th Vehicular Technology Conference (VTC-Fall), Toronto, Canada, Sep. 2017, pp. 1-5.

[20] Z. Wei, Z. Guo, Z. Feng, J. Zhu, C. Zhong, Q. Wu, and H. Wu, "Spectrum sharing between UAV-based wireless mesh networks and ground networks," in 2018 10th International Conference on Wireless Communications and Signal Processing (WCSP), Oct. 2018, pp. 1-6.

[21] C.-H. Yu, K. Doppler, C. B. Ribeiro, and O. Tirkkonen, "Resource sharing optimization for device-to-device communication underlaying cellular networks," IEEE Trans. Wirel. Commun., vol. 10, no. 8, pp. 2752-2763, Aug. 2011.

[22] P. Phunchongharn, E. Hossain, and D. I. Kim, "Resource allocation for device-to-device communications underlaying LTE-advanced networks," IEEE Wirel. Commun., vol. 20, no. 4, pp. 91-100, Aug. 2013.

[23] D.Feng et al, "Device-to- device communications underlaying cellular networks," IEEE Trans. Commun., vol. 61, no. 3541-3551, p. 8, Aug. 2013.

[24] L. Wei, R. Hu, Y. Qian, and G. Wu, "Enable device-to-device communications underlaying cellular networks: challenges and research aspects," IEEE Commun. Mag., vol. 52, no. 6, pp. 90-96, Jun. 2014.

[25] N. Lee, X. Lin, J. G. Andrews, and R. W. Heath, "Power control for D2D underlaid cellular networks: Modeling, algorithms, and analysis," IEEE J. Selec. Areas Commun., vol. 33, no. 1, pp. 1-13, Jan. 2015.

[26] A. Asadi, Q. Wang, and V. Mancuso, "A survey on device-to-device communication in cellular networks," IEEE Commun. Surveys Tuts. vol. 16, no. 4, pp. 1801-1819, Apr. 2014.

[27] G. Yu, L. Xu, D. Feng, R. Yin, G. Y. Li, and Y. Jiang, "Joint mode selection and resource allocation for device-to-device communications," IEEE Trans. Commun., vol. 62, no. 11, pp. 3814-3824, Nov. 2014.

[28] G. George, R. K. Mungara, and A. Lozano, "An analytical framework for device-to-device communication in cellular networks," IEEE Trans. Wirel. Commun., vol. 14, no. 11, pp. 6297-6310, Nov. 2015.

[29] J. Liu, N. Kato, J. Ma, and N. Kadowaki, "Device-to-device communication in LTE-advanced networks: A survey," IEEE Commun. Surveys Tuts., vol. 17, no. 4, pp. 1923-1940, Dec. 2015.

[30] G. Ding, J. Wang, Q. Wu, Y.-D. Yao, F. Song, and T. A. Tsiftsis, "Cellular-base-station-assisted device-to-device communications in TV white space," IEEE J. Select. Areas Commun., vol. 34, no. 1, pp. 107121, Jan. 2016.

[31] D. Zhu, J. Wang, A. Lee Swindlehurst, and C. Zhao, "Downlink resource reuse for device-to-device communications underlaying cellular networks," IEEE Signal Process. Lett., vol. 21, no. 5, pp. 531-534, May 2014.

[32] G. Yu et al, "Joint mode selection and resource allocation for deviceto-device communications," IEEE Trans. Commun., vol. 62, no. 11, pp. 3814-3824, Nov. 2014

[33] K. Yang, S. Martin, C. Xing, J. Wu, and R. Fan, "Energy-efficient power control for device-to-device communications," IEEE J. Selec. Areas Commun., vol. 34, no. 12, pp. 3208-3220, Dec. 2016.

[34] M. Mozaffari, W. Saad, M. Bennis, and M. Debbah, "Unmanned aerial vehicle with underlaid device-to-device communications: Performance and tradeoffs," IEEE Trans. Wirel. Commun., vol. 15, no. 6, pp. 39493963, Jun. 2016.

[35] H. H. Kha, H. D. Tuan, and H. H. Nguyen, "Fast global optimal power allocation in wireless networks by local d.c. programming," IEEE Trans. Wireless Commun, vol. 11, no. 2, pp. 510-515, Feb. 2012.

[36] Z. Xue, J. Wang, G. Ding, Q. Wu, Y. Lin, and T. A. Tsiftsis, "Device-todevice communications underlying UAV-supported social networking," IEEE Access, vol. 6, pp. 34 488-34 502, 2018.

[37] W. Huang, Z. Yang, C. Pan, L. Pei, M. Chen, M. Shikh-Bahaei, M. Elkashlan, and A. Nallanathan, "Joint power, altitude, location and bandwidth optimization for UAV with underlaid D2D communications," IEEE Wirel. Commun. Lett., vol. 8, no. 2, pp. 524-527, Feb. 2019.
[38] M. Asadpour, B. van den Bergh, D. Giustiniano, K. Hummel, S. Pollin, and B. Plattner, "Micro aerial vehicle networks: An experimental analysis of challenges and opportunities," IEEE Commun. Mag., vol. 52, no. 7, pp. 141-149, 2014

[39] Z. Sheng, H. D. Tuan, A. A. Nasir, T. Q. Duong, and H. V. Poor, "Power allocation for energy efficiency and secrecy of wireless interference networks," IEEE Trans. Wirel. Commun., vol. 16, no. 7, pp. 3737-3751, Jun. 2018.

[40] H. Tuy, Convex Analysis and Global Optimization (Second edition). Springer, 2017.

[41] E. Che, H. D. Tuan, and H. H. Nguyen, "Joint optimization of cooperative beamforming and relay assignment in multi-user wireless relay networks," IEEE Trans. Wirel. Commun., vol. 13, no. 10, pp. 5481-5495, Oct. 2014.

[42] H. H. M. Tam, H. D. Tuan, D. T. Ngo, T. Q. Duong, and H. V. Poor, "Joint load balancing and interference management for small-cell heterogeneous networks with limited backhaul capacity," IEEE Trans. Wirel. Commun., vol. 16, no. 2, pp. 872-884, Feb. 2017.

[43] D. Peaucelle, D. Henrion, and Y. Labit, "Users guide for SeDuMi interface $1.03, " 2002$

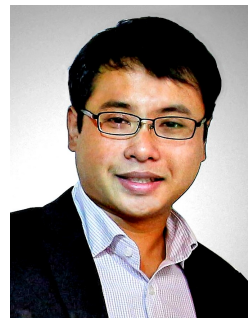

Huy T. Nguyen received the B.S. degree (Hons.) in Computer Science and Engineering from HCMC University of Technology, Vietnam, in 2013. He received the M.S. degree and Ph.D. degree from the Department of Information and Communication System, Inje University, South Korea in 2016, and 2019, respectively. He is currently a Research Fellow at Nanyang Technological University (NTU), Singapore. He was a visiting student with Queen's University of Belfast, U.K. in 2017 and internship $\mathrm{Ph} . \mathrm{D}$ student in National Institute of Information and Communications Technology (NICT), Japan in 2018. His research interests include performance analysis and optimization techniques in signal processing for wireless communications.

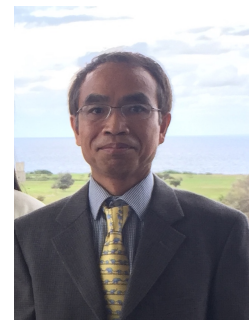

Hoang Duong Tuan received the Diploma (Hons.) and $\mathrm{Ph} . \mathrm{D}$. degrees in applied mathematics from Odessa State University, Ukraine, in 1987 and 1991, respectively. He spent nine academic years in Japan as an Assistant Professor in the Department of Electronic-Mechanical Engineering, Nagoya University, from 1994 to 1999, and then as an Associate Professor in the Department of Electrical and Computer Engineering, Toyota Technological Institute, Nagoya, from 1999 to 2003. He was a Professor with the School of Electrical Engineering and Telecommunications, University of New South Wales, from 2003 to 2011. He is currently a Professor with the School of Electrical and Data Engineering and a Core Member of the Global Big Data Technologies Centre, University of Technology Sydney. He has been involved in research with the areas of optimization, control, signal processing, wireless communication, and biomedical engineering for more than 20 years. 


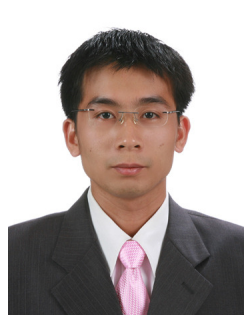

Trung Q. Duong (S'05, M'12, SM'13) received his $\mathrm{Ph} . \mathrm{D}$. degree in Telecommunications Systems from Blekinge Institute of Technology (BTH), Sweden in 2012. Currently, he is with Queen's University Belfast (UK), where he was a Lecturer (Assistant Professor) from 2013 to 2017 and a Reader (Associate Professor) from 2018. His current research interests include wireless communications, machine learning, realtime optimisation, big data, and IoT applications to disaster management, airquality monitoring, flood monitoring, smart agriculture, healthcare and smart cities. He is the author or co-author of over $350+$ technical papers published in scientific journals (210+ articles) and presented at international conferences (140+ papers).

Dr. Duong currently serves as an Editor for the IEEE TRANSACTIONS ON Wireless COMMUNICATIONS, IEEE TRANSACTIONS ON COMMUNICATIONS, and a Lead Senior Editor for IEEE COMMUNiCATIONS LETTERS. He was awarded the Best Paper Award at the IEEE Vehicular Technology Conference (VTC-Spring) in 2013, IEEE International Conference on Communications (ICC) 2014, IEEE Global Communications Conference (GLOBECOM) 2016 and 2019, IEEE Digital Signal Processing Conference (DSP) 2017, and International Wireless Communications \& Mobile Computing Conference (IWCMC) 2019. He is the recipient of prestigious Royal Academy of Engineering Research Fellowship (2016-2021) and has won a prestigious Newton Prize 2017.

H. Vincent Poor (S'72, M'77, SM'82, F'87) received the Ph.D. degree in EECS from Princeton University in 1977. From 1977 until 1990, he was on the faculty of the University of Illinois at UrbanaChampaign. Since 1990 he has been on the faculty at Princeton, where he is currently the Michael Henry Strater University Professor of Electrical Engineering. During 2006 to 2016, he served as Dean of Princeton's School of Engineering and Applied Science. He has also held visiting appointments at several other universities, including most recently at Berkeley and Cambridge. His research interests are in the areas of information theory, signal processing and matching learning, and their applications in wireless networks, energy systems and related fields. Among his publications in these areas is the recent book Multiple Access Techniques for $5 G$ Wireless Networks and Beyond. (Springer, 2019).

Dr. Poor is a member of the National Academy of Engineering and the National Academy of Sciences, and is a foreign member of the Chinese Academy of Sciences, the Royal Society, and other national and international academies. Recent recognition of his work includes the 2017 IEEE Alexander Graham Bell Medal and a D.Eng. honoris causa from the University of Waterloo awarded in 2019.

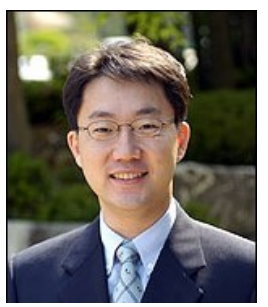

Won-Joo Hwang (M'03, SM'17) received the Ph.D. Degree in Information Systems Engineering from Osaka University Japan in 2002. He received his B.S. and M.S. degree in Computer Engineering from Pusan National University, Pusan, Republic of Korea, in 1998 and 2000. He is now a full professor at Inje University, Gyeongnam, Republic of Korea. His research interests are in network optimization and cross layer design. Dr. Hwang is a member of the IEICE and IEEE. 